Acta Geotechnica manuscript No.

(will be inserted by the editor)

\title{
Liquefaction analysis and damage evaluation of embankment-type structures
}

\author{
Ioanna Rapti · Fernando \\ Lopez-Caballero · Arezou
}

Modaressi-Farahmand-Razavi .

Alexandre Foucault • Francois Voldoire

Received: date / Accepted: date

\begin{abstract}
The increasing importance of performance-based earthquake engineering analysis points out the necessity to assess quantitatively the risk of liquefaction of embankment-type structures. In this extreme scenario of soil I. Rapti · F. Lopez-Caballero · A. Modaressi-Farahmand-Razavi Laboratoire MSS-Mat CNRS UMR 8579, CentraleSupélec Paris-Saclay University, 91190 Gif-Sur-Yvette, France

E-mail: $\quad$ ioanna.rapti@ecp.fr, fernando.lopez-caballero@centralesupelec.fr, arezou.modaressi@centralesupelec.fr

I. Rapti · A. Foucault · F. Voldoire

Institut des Sciences de la Mécanique et Applications Industrielles, EDF-CNRS-CEAENSTA UMR 9219, 7 boulevard Gaspard Monge, 91120 Palaiseau France

E-mail: ioanna.rapti@edf.fr, alexandre.foucault@edf.fr, francois.voldoire@edf.fr

I. Rapti · A. Foucault · F. Voldoire

EDF R\&D Division, 7 boulevard Gaspard Monge, 91120 Palaiseau France
\end{abstract}


liquefaction, devastating consequences are observed, e.g. excessive settlements, lateral spreading and slope instability. The present work discusses the global dynamic response and interaction of an earth structure-foundation system, so as to determine quantitatively the collapse mechanism due to foundation's soil liquefaction. A levee-foundation system is simulated and the influence of characteristics of input ground motion, as well as, of the position of liquefied layer on the liquefaction-induced failure is evaluated. For the current levee model, its induced damage level (i.e. induced crest settlements) is strongly related to both liquefaction apparition and dissipation of excess pore water pressure on the foundation. The respective role of input ground motion characteristics is a key component for soil liquefaction apparition, as long duration of mainshock can lead to important nonlinearity and extended soil liquefaction. A circular collapse surface is generated inside the liquefied region and extends towards the crest in both sides of the levee. Even so, when the liquefied layer is situated in depth, no significant effect on the levee response is found. This research work provides a reference case study for seismic assessment of embankment-type structures subjected to earthquake and proposes a high-performance computational framework accessible to engineers.

Keywords Dynamic analysis · Nonlinear coupled hydromechanical behavior . Soil liquefaction · Earthquake loading $\cdot$ strain localization · instability $\cdot$ FE modeling 


\section{Introduction}

Liquefaction-induced failure of earth structures, such as river dikes, levees, road embankments and earth dams, is identified as one of the most devastating consequences of earthquakes. Previous numerical or experimental studies (e.g. centrifuge tests) have shown that the widespread damage to such embankments occurred mainly due to the liquefaction of foundation soil, resulting in excessive settlements, lateral spreading and slope instability (Aydingun and Adalier, 2003; Stamatopoulos and Aneroussis, 2004; Singh et al, 2005; Oka et al, 2012; Maharjan and Takahashi, 2014; Ishikawa et al, 2015; LopezCaballero et al, 2016). In most cases of foundation's liquefaction, important excess pore pressure and correlated shear strains are generated in the liquefied region and extended inside the geostructures. The shear zones, combined with large settlements and eventual lateral spreading, may lead to local failure or complete collapse of the earthen structures (Sasaki and Tamura, 2007; Okamura et al, 2013; Sadeghi et al, 2014; Ishikawa et al, 2015). Moreover, the influence of foundation soil type and earthquake's characteristics have proven to be critical (Ozutsumi et al, 2002; Adalier and Sharp, 2004; Xia et al, 2010; Lanzo and Pagliaroli, 2012; Maharjan and Takahashi, 2014).

Consequently, the current work describes the dynamic response of a leveefoundation system and focuses on the identification of the liquefaction-induced failure path. It can be considered as a reference case-study for seismic assessment of embankment-type structures subjected to earthquake loading. Parametric studies are conducted to explore the influence of the depth of the liq- 
uefiable layer and of the characteristics of the input motion on the collapse mechanism. For this reason, a plane-strain FE model of a levee-foundation system is built to analyze the liquefaction-induced failure modes. The nonlinear soil behavior is represented using the fully coupled effective stress ECP constitutive model developed at CentraleSupélec (Hujeux, 1985). Numerical simulations are performed using the open-source FE software Code_Aster , developed by EDF (http://www.code-aster.org/).

The first sections are dedicated to the description of the general background of the dynamic analysis, focused on liquefaction-induced failure and of the ECP soil constitutive model. Next, the numerical model used is presented and the typical dynamic response of the levee-foundation system is discussed, i.e. identification of onset of failure path and structure's stability. Furthermore, the effect of the depth of the liquefiable layer on the response is assessed. Finally, as a first effort of liquefaction vulnerability analysis, the global response of the system subjected to different types of seismic excitation is provided, so as to assess the effect of the input motion. At the last section, the concluding remarks regarding the failure path are summarized based on the findings of the study.

\section{Background of dynamic liquefaction analysis}

The main objective of this work is to analyze the dynamic response of embankment type structures and more precisely evaluate the effects of pore water pressure generation and possible liquefaction apparition in the soil foundation on 
the induced damage. In this section, a coherent methodology is proposed and the different steps followed in order to achieve the objective set as explained.

In the literature many parameters are used to define the occurrence of liquefaction, namely, the excess pore water pressure ratio $\left(r_{u}=\Delta p_{w} / \sigma_{v, 0}^{\prime}\right)$ or the effective stress decreasing ratio $\left(\mathrm{ESDR}=1-\sigma_{m}^{\prime} / \sigma_{m 0}^{\prime}\right)$ (Ozutsumi et al, 2002; Lu et al., 2008) among others. Where $\Delta p_{w}$ is the excess pore water pressure, $\sigma_{v, 0}^{\prime}$ is the initial effective vertical stress calculated after the construction of the embankment, the mean effective stress $\left(\sigma_{m}^{\prime}=\left(\sigma_{h, \text { in }}^{\prime}+\sigma_{h, \text { out }}^{\prime}+\sigma_{v}^{\prime}\right) / 3\right.$, with $\sigma_{h, \text { in }}^{\prime}$ and $\sigma_{h, \text { out }}^{\prime}$ the effective in-plane and out-of-plane horizontal stresses respectively). Figure 1 summarizes a comparison of the evolution of $\Delta p_{w} / \sigma_{v 0}^{\prime}$ and $1-\sigma_{m}^{\prime} / \sigma_{m 0}^{\prime}$ for two typical cases. It is noted that from a practical point of view, the obtained evolution using the two ways to define the liquefaction provide a similar response.

In the following, the parameter $r_{u}$ will be used to measure the pore water pressure evolution and potentially occurrence of "liquefaction". Even if in some cases, the $r_{u}$ parameter can exceed 1.0 because of the increase of the vertical stress due to induced vertical accelerations for example, the choice of this parameter does not constitute a loss of generality of the proposed methodology. Then, so as to take into account both "true liquefaction" (i.e. $r_{u}=1.0$ ) and "cyclic mobility" (i.e. $0.7<r_{u}<1.0$ with development of large strains), it is assumed that liquefaction appears when $r_{u}$ is greater than 0.8 (Koutsourelakis et al., 2002; Popescu et al., 2006). 


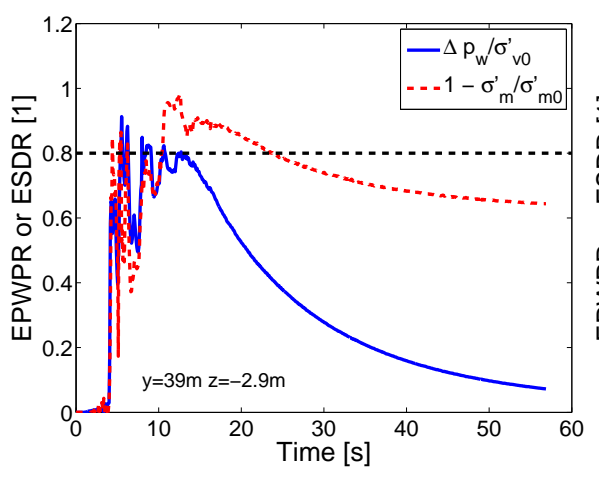

(a)

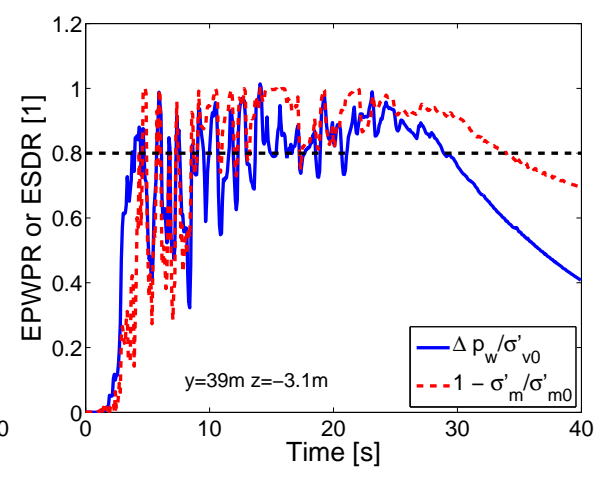

(b)

Fig. 1: Time histories of ESDR and $r_{u}$ evolution for two different input earthquakes..

Once the liquefaction is detected, the deformed shape of the structure is observed in order to identify its failure path. For this purpose the work of Sasaki and Tamura (2007) is employed, which discusses the failure type of river dikes subjected to earthquakes according to the classification of damage modes provided by the "Manual for repair methods of the civil engineering structures damaged by earthquakes" (Technical Note of PWRI, Vol. 45, 1986). Based on this manual, embankment failure due to earthquakes is classified into 4 fundamental modes as shown in Figure 2. Type 1 and 2 refer to the failure in the embankment, type 3 is intense deformation of embankment due to soil liquefaction in the foundation, and type 4 is crest settlement without apparent deformation of the whole embankment.

Moreover, since strain localization is identified as a potential earthquakeinduced failure mode, the deviatoric strains are used to represent earthquake- 


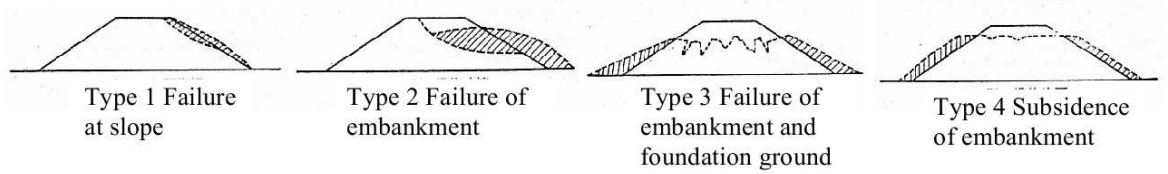

Fig. 2: Classification of damage modes of failed dikes (Sasaki and Tamura, 2007).

induced shear bands and are calculated as:

$$
\varepsilon_{d}=\sqrt{\frac{2}{3} \epsilon_{i j}: \epsilon_{i j}}
$$

where $\epsilon_{i j}=\varepsilon_{i j}-\frac{1}{3} \operatorname{tr}(\varepsilon) \delta_{i j}$. However, in most cases of liquefaction, the failure is often of diffuse type and characterized by the absence of localization and consequently, other approaches are necessary to take into account this mode of failure, i.e. earthquake-induced instability and soil's residual strength, as explained below.

Thus, after the identification of the collapse mechanism, the stability of the structure is investigated. It is widely accepted that liquefaction is an instability and it can occur under static or dynamic conditions. Several researchers have shown the unstable behavior of the material and proposed instability criteria for defining the onset of soil liquefaction (Lade, 1994; Borja, 2006; Zhang and Wang, 2012; Andrade et al, 2013; Mohammadnejad and Andrade, 2015; Najma and Latifi, 2017). Throughout this work, the levee's stability is calculated based on Hill's instability criterion of the second order work (Hill, 1958; Hamadi et al, 2008; Buscarnera and di Prisco, 2012). The instability is described locally 
by Hill's sufficient instability condition for the elasto-plastic materials with associated plastic strains, and by Bigoni and Hueckel (1991) condition for the non-associative elasto-plastic materials. These conditions are related to the sign of the second order work and allow a local follow-up of instabilities. This condition is expressed as follows: A stress-strain state is called "stable", if for any incremental stress and strain linked by the constitutive relation the second order work is strictly positive (Hamadi et al, 2008):

$$
\mathrm{d}^{2} \mathrm{~W}=\mathrm{d} \sigma_{i j} \mathrm{~d} \varepsilon_{i j}>0, \forall(\underline{\underline{\mathrm{d}}}(\mathrm{d} \underline{\underline{\varepsilon}}), \mathrm{d} \underline{\underline{\varepsilon}})
$$

For the current analysis the normalized second-order work is calculated using the effective stresses $\sigma_{i j}^{\prime}$, as considered by Darve and Laouafa (2000):

$$
\mathrm{d}^{2} \mathrm{~W}=\frac{\mathrm{d} \sigma_{i j}^{\prime} \mathrm{d} \varepsilon_{i j}}{\left\|\mathrm{~d} \sigma_{i j}^{\prime}\right\|\left\|\mathrm{d} \varepsilon_{i j}\right\|}, \forall\left(\mathrm{d} \underline{\underline{\sigma^{\prime}}}(\underline{\mathrm{d}} \underline{\underline{\varepsilon}}), \mathrm{d} \underline{\underline{\varepsilon}}\right)
$$

However, in cases of cyclic loading this criterion is not cumulative and gives only an instantaneous instability at specific instants of the ground motion and specific locations.

Finally, intending to observe co-seismic and post-seismic response, the soil's residual strength is calculated, through the parameter $r_{a p t}$ which represents the "distance to reach the critical state", as will be further explained in the next section. The ratio of apparent to critical friction angle provides a reliable measure of soil's strength and accounts for the changes in the material behavior during cyclic loading. For the scope of this work, it is used as a criterion for estimating the local state of soil and quantify local safety factor. 


\section{Soil constitutive model}

The ECP elastoplastic multi-mechanism constitutive model (Hujeux, 1985; Lopez-Caballero and Modaressi-Farahmand-Razavi, 2008) is used to represent soil behavior under cyclic loading and implemented into Code_Aster (Foucault, 2009) using an implicit direct time integration algorithm. This model can account for soil behavior in a large range of loading paths. The representation of all irreversible phenomena is idealized by four coupled elementary plastic mechanisms: three plane-strain deviatoric plastic strain mechanisms in three orthogonal planes ( $k$-planes, with $k=1,2,3)$ and an isotropic one $(k=4)$. The model uses a Coulomb-type failure criterion and the critical state concept. The evolution of hardening is based on plastic strain (deviatoric and volumetric strain for the deviatoric mechanisms and volumetric strain for the isotropic one). To account for cyclic behavior, both isotropic and kinematical hardenings are used. The elasticity domain is isotropic and nonlinear, where the bulk and shear moduli are functions of the mean effective stress. Elastic mechanical characteristics $E, \nu$ must also be defined. Some details about the ECP model are provided in annexe A and in Lopez-Caballero and ModaressiFarahmand-Razavi (2008) among others. For the sake of brevity only some model definitions are given in what follows.

Adopting the soil mechanics sign convention (compression positive), the deviatoric primary yield surface of the $k$ plane is given by:

$$
f_{k}\left(\sigma^{\prime}, \varepsilon_{v}^{p}, r_{k}\right)=q_{k}-\sin \phi_{p p}^{\prime} \cdot p_{k}^{\prime} \cdot F_{k} \cdot r_{k}
$$


where $p_{k}^{\prime}$ and $q_{k}$ are the effective mean and deviatoric values of stress tensor projected on the $k$ plane, $\phi_{p p}^{\prime}$ is the friction angle at critical state, the function $F_{k}$ controls the isotropic hardening associated with the plastic volumetric strain, whereas $r_{k}$ accounts for the isotropic hardening generated by plastic shearing. They represent progressive friction mobilization in the soil and their product reaches unity at perfect plasticity.

Therefore, in order to provide for any state a direct measure of "distance to reach the critical state" $\left(r_{a p t}\right)$ and based upon the used elastoplastic model, it is possible to define an apparent friction angle $\left(\phi_{\text {apt }}^{\prime}\right)$ as follows (Lopez-Caballero and Modaressi-Farahmand-Razavi, 2013):

$$
\begin{aligned}
\sin \phi_{a p t}^{\prime} & =\frac{q_{k}}{p_{k}^{\prime} \cdot F_{k}} \\
r_{a p t} & =\frac{\sin \phi_{a p t}^{\prime}}{\sin \phi_{p p}^{\prime}}
\end{aligned}
$$

It varies between 0 and 1 where perfect plasticity is reached and is defined as the inverse of a local safety factor $\left(r_{a p t}=1 / \mathrm{FS}\right.$, i.e. near collapse when $\left.r_{a p t}=\mathrm{FS}=1.0\right)$. Note that in case of monotonic loading, the parameter $r_{a p t}$ is identical to $r_{k}$ of the $k$ th plane.

\section{Model description}

The FE analysis is performed in three steps: a) as the model is nonlinear, a static analysis is performed in order to calculate the initial stresses under gravity loading, b) the levee is constructed by layers (50 cm/2days: total 36 days), c) a model initialization is realized by enforcing zero displacements, so as to 
account for the co-seismic displacements only and d) the final step is the application of the seismic signal.

\subsection{Geometry}
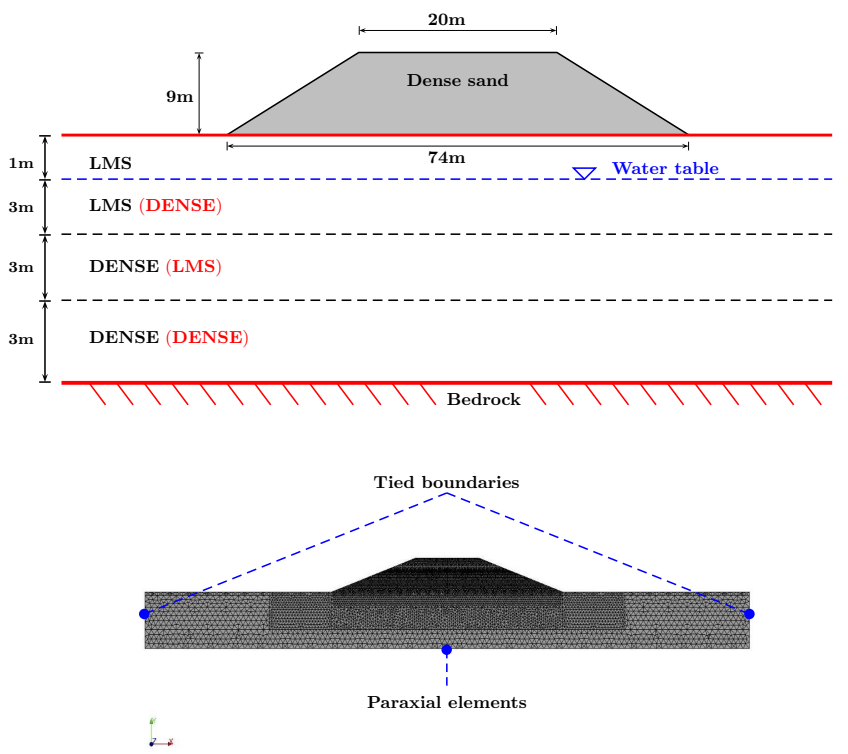

Fig. 3: Illustration of numerical model and used mesh.

The $9 \mathrm{~m}$ high levee is composed of a dry dense sand, placed over a loose-tomedium contractive saturated sand (LMS) layer. The levee remains always dry and the water table is situated $1 \mathrm{~m}$ below the levee. The levee's slope inclination is equal to $1: 3$ (vertical:horizontal).

Firstly, the liquefiable layer (LMS layer) is supposed to be close to the free surface, as shown in Figure 3 with black color, and a dense saturated substratum exists at the bottom part of the foundation. Then, in order to assess 
the effect of the depth of the liquefied region on levee's response, the liquefiable layer is situated deeper, between two layers of dense sand, as presented in Figure 3 with red color. In both cases, an elastic rigid bedrock $\left(\mathrm{V}_{s}=1000 \mathrm{~m} / \mathrm{s}\right)$ is simulated below the dense substratum.

\subsection{Boundary conditions}

For the construction phase, horizontal displacements are fixed at the lateral boundaries, as well as vertical displacements at the bedrock. However, for the dynamic analysis, vertically incident shear waves are introduced into the domain and as the response of an infinite semi-space is modelled, equivalent boundaries have been imposed on the nodes of lateral boundaries (i.e. the normal stress on these boundaries remains constant and the displacements of nodes at the same depth in two opposite lateral boundaries are the same in all directions). The model length ensures that the effect of the boundaries has a very low influence on the behaviour of the levee and it satisfies the free field condition at the lateral boundaries. For the half-space bedrock's boundary condition, paraxial elements simulating "deformable unbounded elastic bedrock" have been used (Modaressi and Benzenati, 1994). The incident waves, defined at the outcropping bedrock are introduced into the base of the model after deconvolution. Thus, the obtained movement at the bedrock is composed of the incident waves and the diffracted signal. 
4.3 Soil behavior

The dry levee is composed of a dilative dense sand, while the foundation is composed of a contractive loose-to-medium sand (LMS) and dense sand. Their parameters can be found in Table 3 and were determined with the procedure defined by Lopez-Caballero et al (2007), for their calibration and validation refer to Costa D'Aguiar et al (2011) and Saez (2009). The soil parameters of the dense sand of the levee are given by Nguyen (2006).

In order to understand the behavior of the chosen materials under dynamic loading, cyclic shear drained and triaxial cyclic undrained tests are conducted. Figure 4a shows $G / G_{\max }-\gamma$ curves for the dense sand of the levee and the LMS of the foundation under drained cyclic shear tests at confining pressure corresponding to the average geostatic pressure of the levee, i.e. $\mathrm{p}_{0}=50 \mathrm{kPa}$ and for a coefficient of lateral earth pressure $K_{0}=1.0$. The obtained curves are in good agreement with the reference curves given by Seed and Idriss (1970). The liquefaction curve for the LMS is provided in Figure $4 \mathrm{~b}$ after triaxial cyclic undrained test. The results match relatively good with the experimental ones provided by Byrne et al (2004), which correspond to the liquefaction response of Nevada sand for a range of relative densities. In addition, Figures $4 \mathrm{c}$ and $4 \mathrm{~d}$ show the response obtained by the model in simulated drained triaxial tests. The response is showed in $q-\varepsilon_{1}$ and $\varepsilon_{v}-\varepsilon_{1}$ planes. 


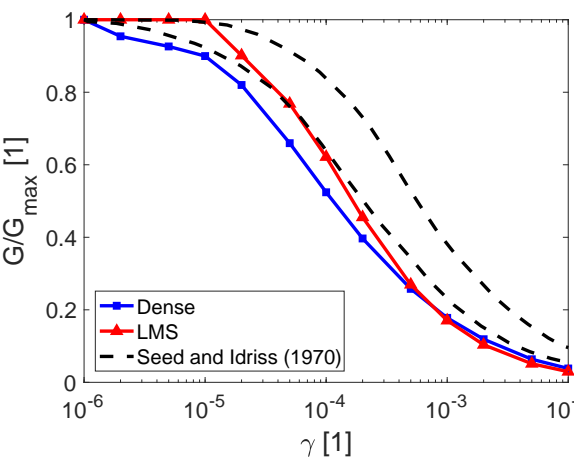

(a) $G / G_{\max }-\gamma$

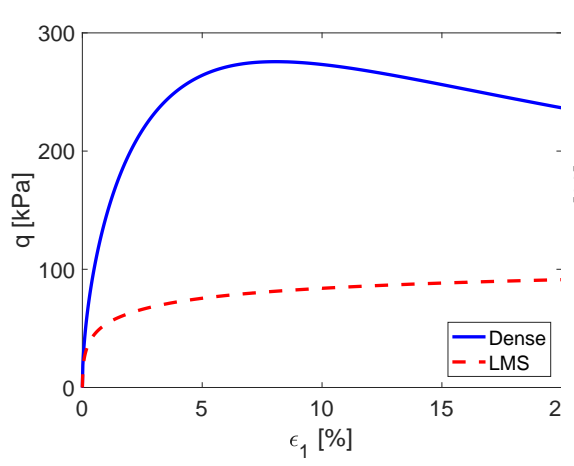

(c) $q-\varepsilon_{1}$

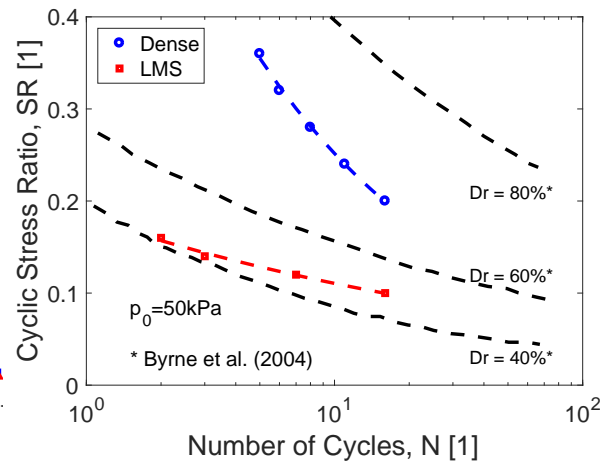

(b) Liquefaction curve

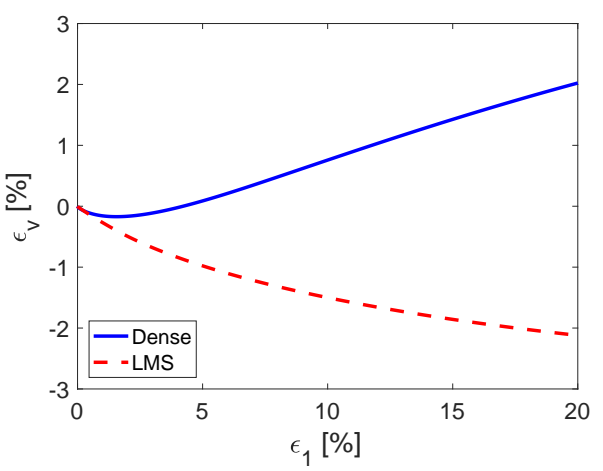

(d) $\varepsilon_{v}-\varepsilon_{1}$

Fig. 4: Soil response of one material point with the ECP constitutive model $\left.\left(\mathrm{p}_{0}=50 \mathrm{kPa}, \mathrm{K}_{0}=1.0\right): \mathrm{a}\right)$ Shear cyclic drained test compared to Seed and Idriss (1970), b) Cyclic triaxial undrained test: Liquefaction curve compared to Byrne et al (2004), c) and d) Simulated drained triaxial tests.

\subsection{Hydraulic behavior}

A fully coupled effective stress dynamic approach using the $\underline{u}-p_{w}$ formulation derived from Biot's theory for incompressible solid-compressible fluid is used, also named Terzaghi's hypothesis (Zienkiewicz et al., 1980; Modaressi, 1987; 
Aubry and Modaressi, 1990; Oka et al, 1994; Zienkiewicz et al, 1990; Coussy, 1991). In the $\underline{u}-p_{w}$ formulation, the displacement of the solid phase $\underline{u}$ and the pore water pressure $p_{w}$ are used as unknown variables. The fluid's relative acceleration with respect to soil skeleton is much smaller than the acceleration of the solid phase and is considered negligible (Zienkiewicz et al, 1990). This formulation is valid for low-frequency phenomena, such as earthquake motions and reduces the total number of degrees of freedom. The governing equations of the coupled dynamic approach consist of the dynamic equilibrium of the soil mixture and the fluid balance equation. In this particular study, in the description of Darcy's law the soil skeleton's acceleration $(\ddot{u})$ is neglected (Granet, 2015) and consequently the governing equations solved in the FE software are:

$$
\begin{array}{r}
\sigma_{i j, j}^{\prime}+p_{w, i}+\rho \cdot F_{i}-\rho \cdot \ddot{u}_{i}=0 \\
-p_{w, i i}-\rho_{w} \cdot \ddot{u}_{i, i}-\rho_{w} \cdot F_{i, i}+\frac{n \cdot \gamma_{w}}{k_{s_{i j}} \cdot K_{w}} \cdot \dot{p}_{w}+\frac{\gamma_{w}}{k_{s_{i j}}} \cdot \dot{u}_{i, i}=0
\end{array}
$$

where $\rho=(1-n) \cdot \rho_{s}+n \cdot \rho_{w}=$ total density (with $\rho_{s}$ solid density, $\rho_{w}$ water density and $n$ porosity), $\sigma_{i j}^{\prime}$ is the Terzaghi's effective stress tensor, $p_{w}$ is the pore water pressure, $F_{i}=$ body forces, $\gamma_{w}=\rho_{w} \cdot \mathrm{g}=$ specific weight of the water, $k_{s_{i j}}$ is the permeability tensor (in $\mathrm{m} / \mathrm{s}$, similar to a velocity), $H_{w}=$ water compressibility (i.e. $H_{w}=1 / K_{w}$, where $K_{w}=-\dot{p}_{w} / \dot{u}_{i, i}^{w}=$ water bulk modulus). The soil skeleton displacement $\underline{u}$ and pore water pressure $p_{w}$ verify the above governing equations, the limit and initial conditions, as well as, the material's constitutive law which was described previously.

So as to accentuate the effect of soil liquefaction, a silty sand of low permeability $\left(k_{s}=1 \cdot 10^{-5} \mathrm{~m} / \mathrm{s}\right)$ is used for the liquefiable layer (Bardet, 1997). This 
characterization is based on the relation of permeability with particle size and void ratio established by NAVFAC (1982) and on the classification of soils after Terzaghi and Peck (1967). The permeability of the dense substratum is considered 10 times lower than this of the liquefiable layer $\left(k_{s}=1 \cdot 10^{-6} \mathrm{~m} / \mathrm{s}\right)$. For the needs of FE modeling, the value of fluid compressibility used, is higher than the real one of water. When the real value of water compressibility $\left(4.5 \cdot 10^{-10}\right.$ $\mathrm{Pa}^{-1}$ ) is used, it is possible that due to the rapid dynamic loading, an abrupt increase in pore water pressure happens and liquefaction occurs, resulting to numerical instabilities problems. The hydraulic parameters of each material are presented in Table 1 .

Table 1: Hydraulic parameters for the soil.

\begin{tabular}{lll}
\hline \hline Parameter & Foundation (LMS) & Foundation (Dense sand) \\
\hline \hline Fluid mass density, $\rho_{w}\left[\mathrm{~kg} / \mathrm{m}^{3}\right]$ & 1000 & 1000 \\
Porosity, $n[1]$ & 0.35 & 0.35 \\
Permeability, $k_{s}[\mathrm{~m} / \mathrm{s}]$ & $1 \cdot 10^{-5}$ & $1 \cdot 10^{-6}$ \\
Fluid compressibility, $H_{w}\left[\mathrm{~Pa}^{-1}\right]$ & $9.35 \cdot 10^{-8}$ & $9.35 \cdot 10^{-8}$ \\
\hline
\end{tabular}

4.5 Numerical parameters

The FE mesh consists of 6 -node triangular elements of $0.50 \mathrm{~m}$ length on average (30054 nodes/17538 elements). The implicit method of Newmark integration is used for the dynamic analysis with a time step equal to $\Delta t=10^{-3}$ s. Since, the model is elastoplastic, no damping exists in the elastic domain and numerical 
damping should be added. According to the generalized $\alpha$-method, the value of damping depends on the time step and frequency (i.e. spectral radius $\rho_{\infty}(\Delta t$, f)) (Kuhl and Crisfield, 1999; Hughes, 2000; Kontoe et al, 2008; Ruiz and Saragoni, 2009; Montoya-Noguera and Lopez-Caballero, 2016). In this case, it was calculated as function of the fundamental frequency and is equal to $\xi$ $=0.2 \%$, as the set of integration parameters used is $\beta=0.31$ and $\gamma=0.61$ (spectral radius $\rho_{\infty}=0.8$ ).

The low-strain frequency analysis provides a fundamental elastic period for the levee equal to $\mathrm{T}_{p}=0.22 \mathrm{~s}$ (i.e. $\mathrm{f}_{p}=4.5 \mathrm{~Hz}$ ). It is obtained from the Borehole Transfer Function from the top to base (i.e. ratio of the frequency response at levee's surface over the bedrock frequency response) for a sample seismic signal at very low amplitude $\left(10^{-6} \mathrm{~g}\right)$ to ensure elastic soil behavior.

4.6 Input ground motions

In order to define appropriate input motions to the non-linear dynamic analysis, a selection of recorded accelerograms are used. The adopted earthquake signals are proposed by Iervolino and Cornell (2005); Baker (2007); Baker et al (2011). Thus, 15 unscaled records were chosen from the Pacific Earthquake Engineering Research Center (PEER) database. The events range between 5.2 and 7.6 in magnitude and the recordings have site-to-source distances from 15 to $50 \mathrm{~km}$ and dense-to-firm soil conditions (i.e. $360 \mathrm{~m} / \mathrm{s}<V_{s} 30 \mathrm{~m}<800 \mathrm{~m} / \mathrm{s}$ ).

The statistics on some input earthquake characteristics obtained for the strong ground motions are summarized in Table 2. These earthquake charac- 
teristics are maximal outcropping acceleration $\left(\mathrm{a}_{\text {out }, \text { max }}\right)$, Arias intensity $\left(I_{A}\right)$, predominant period $\left(T_{p}\right)$, mean period $\left(T_{m}\right)$, peak ground velocity $(P G V)$, period of equivalent harmonic wave $\left(T_{V / A}=\alpha \cdot P G V / a_{\text {out }, \text { max }}\right)$, root-meansquare intensity $\left(I_{r m s}\right)$ and significant duration from $5 \%$ to $95 \%$ Arias intensity $\left(t_{5-95}\right)$. Note that all input and output signals have a baseline correction and are filtered using a non causal 4th-order butterworth bandpass filter, between $0.1-20 \mathrm{~Hz}$

Table 2: Statistics characteristics for the selected earthquakes

\begin{tabular}{lccccc}
\hline Parameter & Range & Mean & $C V[\%]$ & Median & $\sigma_{\ln }$ \\
\hline $\mathrm{a}_{\text {out }, \text { max }}[\mathrm{g}]$ & $0.10-0.70$ & 0.25 & 64 & 0.19 & 0.55 \\
$T_{m}[\mathrm{~s}]$ & $0.11-0.66$ & 0.40 & 38 & 0.42 & 0.47 \\
$T_{p}[\mathrm{~s}]$ & $0.08-0.70$ & 0.22 & 69 & 0.20 & 0.54 \\
$T_{V / A}[\mathrm{~s}]$ & $0.11-0.74$ & 0.38 & 42 & 0.38 & 0.50 \\
$I_{\text {Arias }}[\mathrm{m} / \mathrm{s}]$ & $0.02-3.25$ & 0.69 & 137 & 0.31 & 1.25 \\
$\mathrm{t}_{595}[\mathrm{~s}]$ & $0.91-36.64$ & 9.82 & 95 & 7.24 & 1.01 \\
$I_{\text {rms }}\left[\mathrm{m} / \mathrm{s}^{2}\right]$ & $0.15-0.90$ & 0.34 & 65 & 0.25 & 0.52 \\
$P G V[\mathrm{~cm} / \mathrm{s}]$ & $2.31-50.50$ & 19.18 & 77 & 0.12 & 0.81 \\
\hline
\end{tabular}

\section{Typical dynamic response}

Before analyzing the influence of the aforementioned parameters on the dynamic response of the levee-foundation system, its typical response is presented in case of earthquake loading. The model with the liquefiable layer situated 
close to the surface is used as reference case study and a moderate signal $\left(\mathrm{a}_{\text {out }, \text { max }}=0.24 \mathrm{~g}\right)$ with short duration of mainshock $\left(\mathrm{t}_{595}=0.91 \mathrm{~s}\right)$ is chosen for the current analysis, i.e. EQ2 motion (see Table 4), as shown in Figure 5.

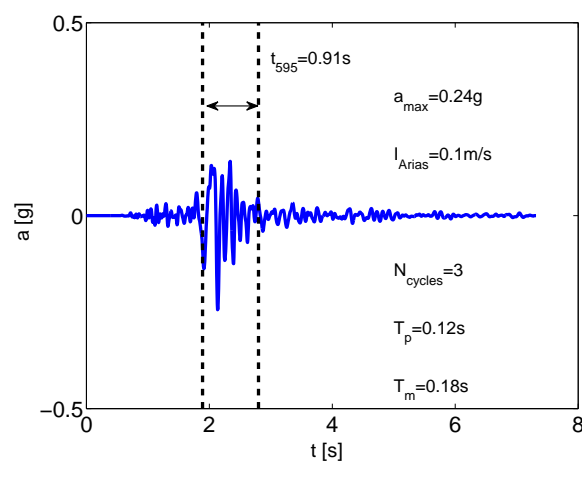

(a)

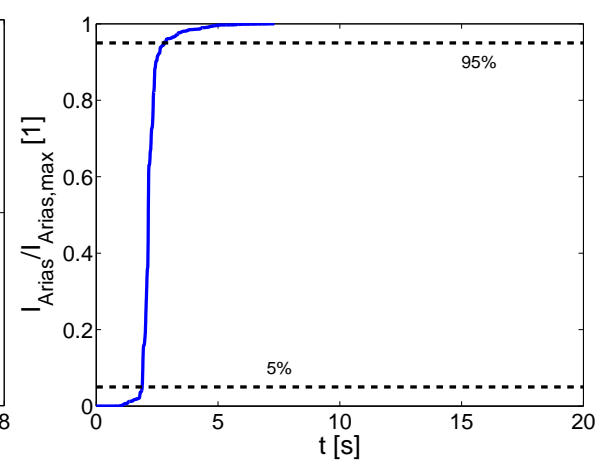

(b)

Fig. 5: Input seismic signal (EQ2): a) Accelerogram, b) Normalized Arias intensity.

As explained previously, the contours of $r_{u}$ are plotted in Figure 6 to identify soil liquefaction. It is noticed that excess pore water pressure is generated in the liquefiable layer of the foundation and lead to liquefaction (i.e. $r_{u}>0.8$ ) below the FF during the mainshock $(\mathrm{t}=2-3 \mathrm{~s})$. The evolution of $\Delta p_{w}$ is related to the mainshock and Arias intensity (Figure $5 \mathrm{~b}$ ), as between $\mathrm{t}=2-3 \mathrm{~s}$ the total intensity of the motion has been accumulated ( $95 \%$ of $\left.\mathrm{I}_{\text {Arias }}\right)$. The liquefaction extended from each toe of the levee to the FF (Free-Field) part. However, low values of $r_{u}$ are observed below the levee due to higher values of stresses and soil's consolidation. After the mainshock, the excess pore water pressure pro- 
gressively dissipates, as the amplitude of the ground motion decreases (coda phase, i.e. last seconds of the ground motion where the amplitude decreases) and the dissipation is completed some seconds after the ground motion.

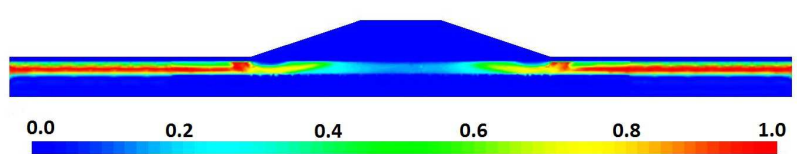

(a) Mainshock ( $\mathrm{t}=2 \cdot 4 \mathrm{~s})$.

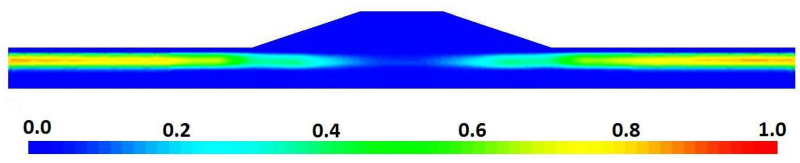

(b) End of earthquake $(t=7.4 \mathrm{~s})$.

Fig. 6: Typical dynamic response (EQ2): Excess of pore water pressure ratio $\left(r_{u}=\Delta p_{w} / \sigma_{o}^{\prime}\right)$.

Due to foundation's liquefaction, a circular slip surface is observed in both sides of the levee (Figure 7). The right and left parts of the levee settle down superficially (Figure 7a) and move towards the FF part (Figure 7b). This failure path is also reported by Sasaki and Tamura (2007), Maharjan and Takahashi (2014) and Ishikawa et al (2015), as lateral spreading combined with settlements. More precisely, as shown in Figure 2, this damage mode can be classified as Type 1, i.e. failure at slope. This type of sliding results from the liquefaction of the foundation and is produced during the mainshock due to the increase of excess pore water pressure. Consequently, no further post- 
liquefaction effects are detected, which is coherent to the results of Coelho et al (2004) after observations in centrifuge tests of saturated sand deposits, where the most significant part of settlement occured simultaneously with the shaking.

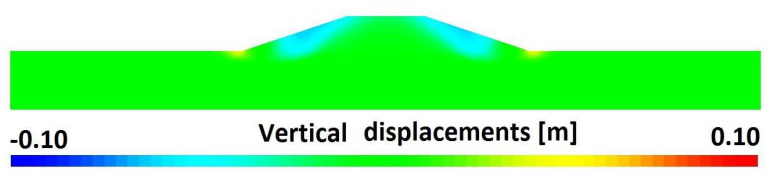

(a) Settlements.

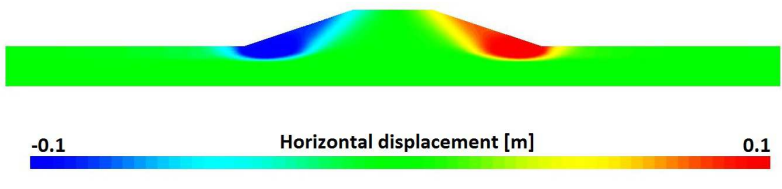

(b) Horizontal displacements.

Fig. 7: Typical dynamic response (EQ2): Deformed shape at the end of the ground motion.

Furthermore, due to foundation's liquefaction, two different shear zones are generated in the liquefied layer below the toes of the levee, as shown in Figure 8 . These shear zones propagate inside the levee body and verify the circular collapse surface identified previously. The generation of shear zones in the foundation is also reported in the research work of Oka et al (2012) focused on the liquefaction-induced failures of river embankments during the Tohoku earthquake in 2011. 


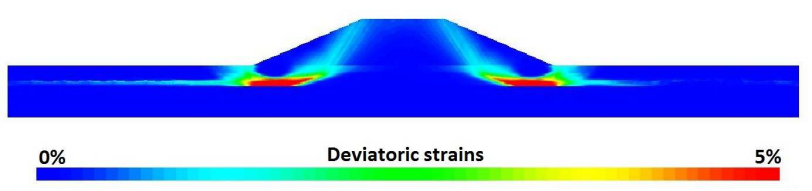

Fig. 8: Typical dynamic response (EQ2): Deviatoric strains $\varepsilon_{d}$ at the end of the ground motion.

In order to verify the soil's behavior in the liquefied region and inside the shear band, one point in each region was selected and the evolution of volumetric - shear strains during the loading is examined. According to Figure 9a, it is noted that two types of responses appear following the onset of liquefaction (Figure 9b). As expected, before the pore water pressure build-up $(\mathrm{t}<2 \mathrm{~s})$ at the beginning of the earthquake, no generation of volumetric deformations is obtained. Then, once the liquefaction has started during the mainshock $(\mathrm{t}=2.2 \mathrm{~s})$, the soil's behavior is purely contractive (blue curve, $\left.\varepsilon_{v}<0\right)$ in the liquefied region of the foundation, meaning that settlements appear. On the other hand, inside the shear band generated in the levee (red curve) the soil becomes dilative $\left(\varepsilon_{v}>0\right)$ after the onset of mainshock $(\mathrm{t}=2.4 \mathrm{~s})$, meaning that the shear band is generated and extends towards the levee and crack opening may occur.

To explore the levee's stability, in Figure 10 the contours of second order work density $\mathrm{d}^{2} \mathrm{~W}$ are presented at $\mathrm{t}=2.4 \mathrm{~s}$ (during the mainshock of the ground motion). The model underwent instability at both sides of the levee and inside the liquefied layer, implying the eventual circular slip surface which 


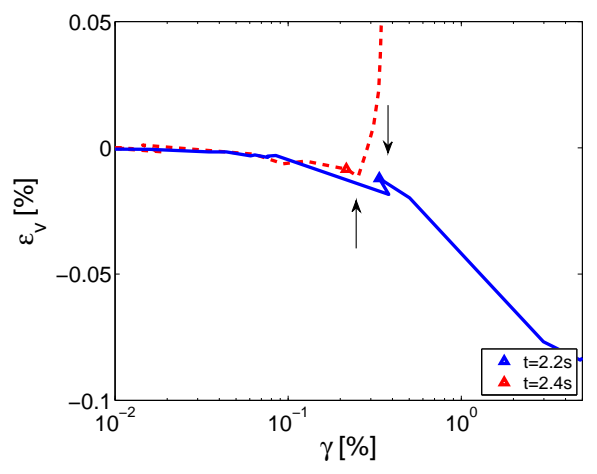

(a)

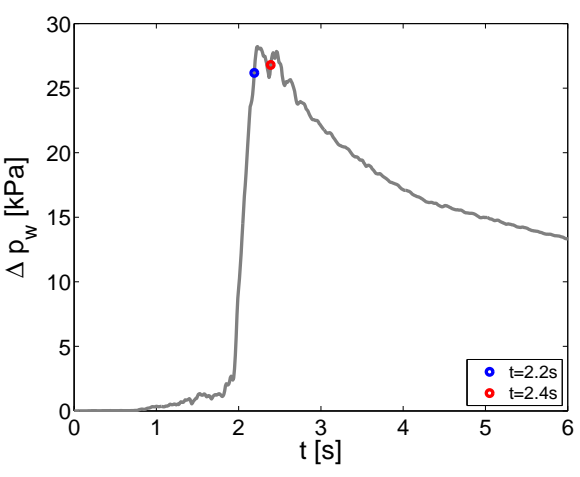

(b)

Fig. 9: Typical dynamic response (EQ2): a) Evolution of volumetric - shear strains during the loading inside the liquefied part and the shear band, b) Evolution of $\Delta p_{w}$ inside the liquefied region.

can lead to the system's collapse. It is observed that these contours corroborate those obtained for the shear strains. At the end of the ground motion and although excess pore water pressures still appear, a stable state is reached. The corresponding figure is omitted as no further information is provided.

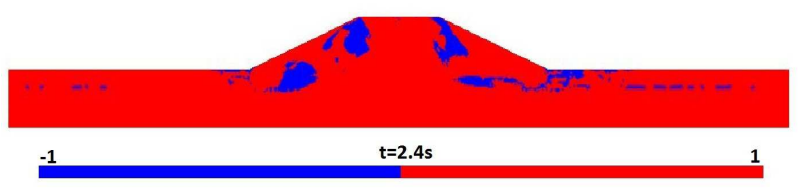

Fig. 10: Typical dynamic response (EQ2): Contours of dimensionless second order work at $\mathrm{t}=2.4 \mathrm{~s}$ (mainshock).

Finally, concerning soil's residual strength, the parameter $r_{a p t}$ is used, as explained previously. In Figure 11a, the contours of $r_{a p t}$ are plotted at the 
beginning of the ground motion and it can be noticed that before the ground motion and due to the construction phase of the levee, noticeable values of $r_{a p t}$ appear in the liquefiable layer below the levee (i.e. $r_{a p t} \simeq 0.6-0.8$ and $\mathrm{FS} \simeq 1.6$ 1.25). In Figure $11 \mathrm{~b}$ during the mainshock $(\mathrm{t}=2.4 \mathrm{~s})$ and while liquefaction is reached $\left(r_{u}>0.8\right)$, the same circular surface of collapse is remarked and $r_{\text {apt }}$ reaches unity (i.e. $\mathrm{FS}=1.0$ ). Even at the end of the motion in Figure 11c important values of $r_{a p t}$ appear inside the zone of shear strains in the foundation, providing a low safety factor of 1.2.

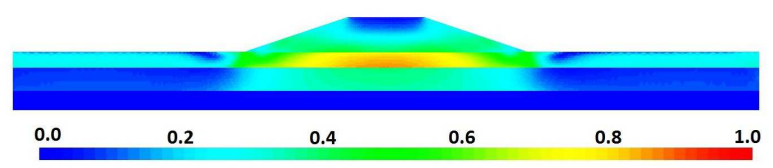

(a) Initial material state $(t=0 s)$.

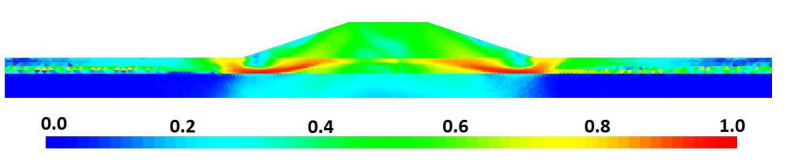

(b) Mainshock $(\mathrm{t}=2 \cdot 4 \mathrm{~s})$.

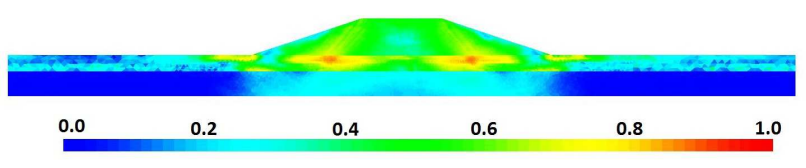

(c) End of earthquake $(t=7.4 \mathrm{~s})$.

Fig. 11: Typical dynamic response (EQ2): Contours of residual strength, $r_{\text {apt }}$.

In the following sections, in order to assess the influence of different parameters on the dynamic response of the levee-foundation system, the results 
of the parametric studies are presented and compared to the typical response. Firstly, the effect of the depth of the liquefied layer is evaluated and then, the influence of the characteristics of the input signal is discussed.

\section{Effect of liquefiable's layer depth}

In this section, the influence of the depth of the liquefiable layer is assessed and the second model indicated with red color in Figure 3 is used. For the sake of brevity, only the results of a strong motion $\left(\mathrm{a}_{\text {out } \max }=0.42 \mathrm{~g}\right)$ with duration of mainshock equal to $t_{595}=4.3 \mathrm{~s}$ are discussed. The accelerogram and Arias intensity of the motion, referred as EQ3 in Table 4, are plotted in Figure 12.

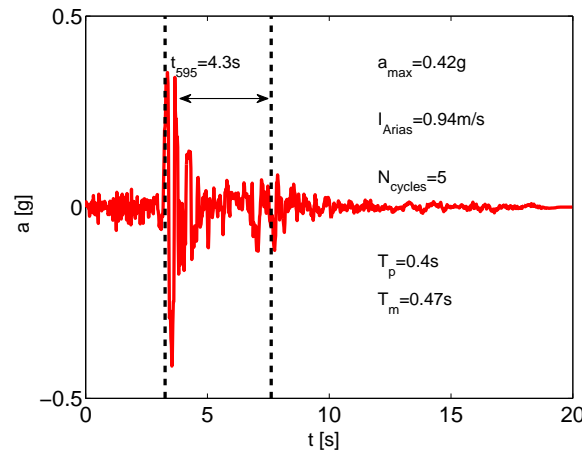

(a)

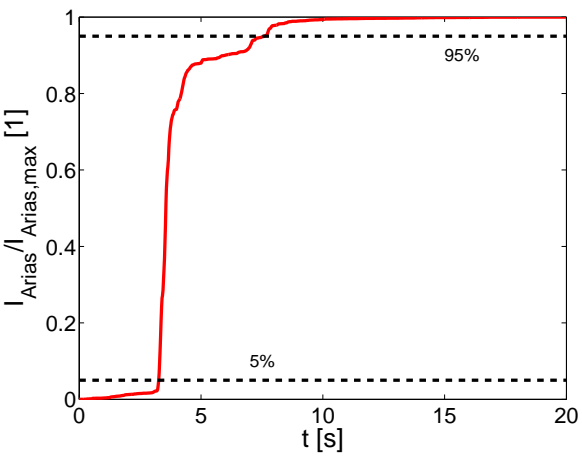

(b)

Fig. 12: Input seismic signal (EQ3): a) Accelerogram, b) Normalized Arias intensity.

Firstly, the contours of $r_{u}$ are plotted in Figure 13 during the mainshock and at the end of earthquake loading. It is observed that excess pore water 
pressure $\left(\Delta p_{w}\right)$ is generated in the liquefiable layer situated in depth in the foundation during the mainshock (Figure 13a). As the motion is strong, liquefaction appears in the whole layer of LMS material in Figure 13a. More in detail, the part below the FF is firstly liquefied with the main peak of the motion and then, excess pore pressure is generated below the levee as the water tries to dissipate. The excess pore water pressure progressively dissipates towards the upper part of the foundation, as increase of $r_{u}$ appears at the end of the mainshock at the denser upper layer $\left(r_{u}=0.6\right.$ in Figure 13b). Compared to the previous model of Figure 6b, the dissipation is much slower in the current model and the excess pore water pressure has not dissipated at the end of the earthquake due to the less permeable layers of dense soil at the upper and lower part of the liquefied layer. The layer of LMS remains liquefied $\left(r_{u}>0.8\right)$ at the end of the earthquake (Figure 13b).

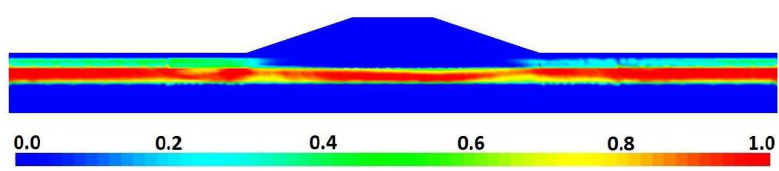

(a) Mainshock $(t=5 \mathrm{~s})$.

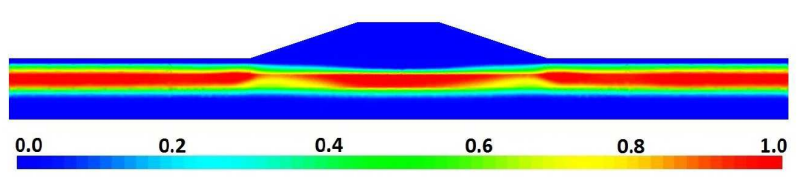

(b) End of earthquake ( $t=20 \mathrm{~s})$.

Fig. 13: Liquefiable layer in depth (EQ3): Excess pore water pressure ratio $\left(r_{u}=\Delta p_{w} / \sigma_{o}^{\prime}\right)$ during the motions. 
The strong earthquake led to noticeable values of settlements that appear all along the levee's crest, as shown in Figure 14a. This damage mode can be classified as Type 4 (Figure 2), i.e. crest settlement without apparent deformation of the whole levee. Furthermore, the foundation moves horizontally towards the FF part at the level of the liquefied layer (Figure 14b) and the displacements are not symmetric, i.e. the left part at the toe of the levee is more affected, probably due to the input signal's asymmetry. It is also important to note that apart from the local instabilities at the liquefied foundation during the motion (as it was also observed previously for the lower motion), at the end of the earthquake instabilities appear inside the levee too, as shown in Figure 15. This can be explained as the foundation remains completely liquefied at the end of the motion and the dissipation of water pressure happens towards the upper soil layers.

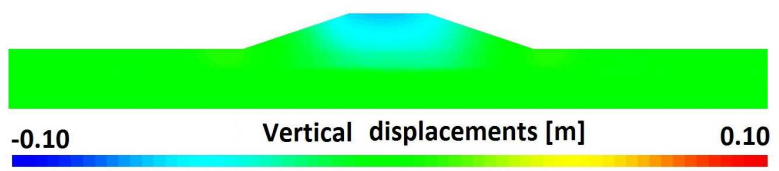

(a) Settlements.

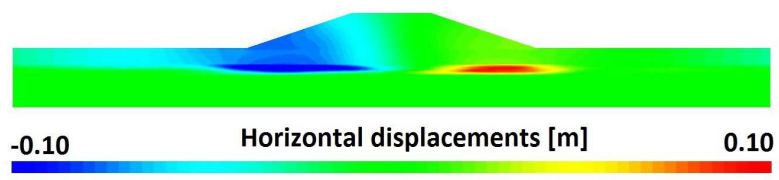

(b) Horizontal displacements.

Fig. 14: Liquefiable layer in depth (EQ3): Deformed shape at the end of the ground motion. 


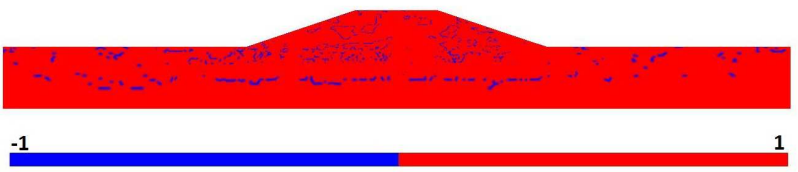

Fig. 15: Liquefiable layer in depth (EQ3): Contours of second order work at the end of earthquake.

Next, concerning the shear strains at the end of the motion, while in the previous model shear zones are generated in the liquefied region and propagate inside the levee body (Figure 8), in the current model the strains are insignificant although the motion imposed is stronger, as shown in Figure 16. As the liquefaction was located in depth, the levee is not affected in terms of shear strains. A diffuse zone of shear strains is observed inside the liquefied layer, but no shear bands propagate to the upper layers. Note that this statement is inextricably related to the characteristics of the ground motion and should not be used as a general conclusion. It is possible that in case of a longer duration of mainshock, more important strains may appear inside the levee, too.

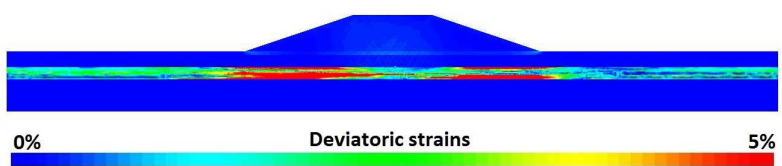

Fig. 16: Liquefiable layer in depth (EQ3): Deviatoric strains $\varepsilon_{d}$ at the end of the ground motion. 
In Figure 17a, the contours of $r_{a p t}$ are plotted at the beginning of the ground motion and as before, noticeable values of $r_{a p t}$ appear in the liquefiable layer due to the construction of the levee (i.e. $r_{a p t} \simeq 0.8$ and $\mathrm{FS} \simeq 1.25$ ). During the ground motion the safety factor remains at acceptable levels and at the end of motion the soil has regained its strength, providing a local safety factor of $1.6\left(r_{a p t}=0.6\right)$, as shown in Figure 17c. High values of residual strength are obtained inside the levee (low value of $r_{a p t} \simeq 0.3$ ), i.e. soil densification, and as expected, it is almost unaffected by the earthquake-induced liquefaction.

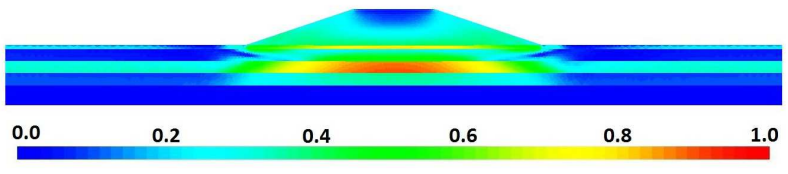

(a) $\mathrm{t}=0 \mathrm{~s}$

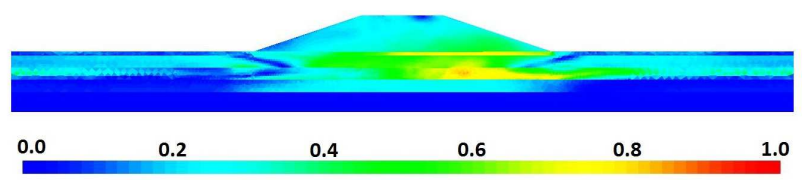

(b) $\mathrm{t}=5 \mathrm{~s}$

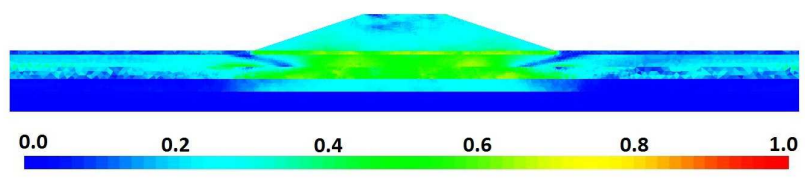

(c) $\mathrm{t}=20 \mathrm{~s}$ (end)

Fig. 17: Liquefiable layer in depth (EQ3): Contours of residual strength, $r_{\text {apt }}$.

Consequent to the aforementioned results, the model with the liquefiable layer situated in depth is less affected and for this reason, in the following 
section the model with the liquefiable layer close to the surface will be used to better observe liquefaction effects.

\section{Effect of earthquake's characteristics on damage analysis}

In the context of Performance-Based Earthquake Engineering of PEER (Porter, 2003), the choice of the input ground motion is crucial for liquefaction vulnerability analysis. In this section a damage analysis of both levee models (Figure 3) subjected to all ground motions is provided and the influence of earthquake's characteristics on liquefaction-induced failure is discussed. In the scope of this research, the liquefaction vulnerability analysis is limited to identifying the IM (Intensity Measure) for the seismic hazard analysis for a given EDP of interest (Engineering Demand Parameter), i.e. the crest settlements. The IM and EDP of interest could be used as input data for the hazard and structural analyses and then proceed to a probabilistic analysis and fragility curves calculation.

Damage level estimation and comparison with an empirical relation

From an engineering point of view, Swaisgood (2003) investigated several dam cases that experienced an earthquake (e.g. dams in Philippines, California, Chile, Peru) and analyzed the database using statistical regression technics in order to identify the factors that have a major influence on the deformation and damage of embankment dams during earthquakes. Different types of dams were considered, but for the purposes of this study only the Hydraulic Fill (HF) 
and Earthfill (E) dams are taken into account. According to this work, crest settlement was selected as the EDP of interest to represent earthquake related deformation because it was the most often mentioned quantified measurement of damage presented in the case histories. It appears to be directly related to the severity of deformation and degradation, i.e. as the percent of crest settlement increases, the extent of deformation and degradation that occurs also increases. Moreover, the PGA experienced by an embankment, as well as the magnitude $(\mathrm{M})$ of the earthquake, had a major influence on the amount of crest settlement and were chosen as representative IMs. It was found that the greater the PGA and M, the greater the deformations and damages. After these observations and statistical studies based on real case histories, ranges of the relative levels of damage were proposed and an empirical relation between crest settlement and PGA and magnitude was established as follows:

$$
\frac{\delta u_{v, \text { crest }}}{H}=\exp (6.07 \cdot P G A+0.57 \cdot M-8) \text { in } \%
$$

where $\delta u_{v, \text { crest }} / \mathrm{H}$ is the percentage of crest settlement of the dam (in $\mathrm{m}$ ) divided by the height $\mathrm{H}$ of the dam plus the thickness of the alluvium (in $\mathrm{m}$ ), PGA is the peak ground acceleration of the foundation rock (in $\mathrm{g}$ ) and $\mathrm{M}$ is the earthquake magnitude (in surface-wave scale, i.e. $\mathrm{M}_{s}$ ). Following the work of Swaisgood (2003), Kim et al (2011) performed centrifuge tests of typical types of Korean dams and the results obtained in terms of recorded settlements matched quite well to the values of field data of Swaisgood (2003) and the proposed damage levels, as the centrifuge results followed a similar trend. 
As a result of the aforementioned work and observations, the damage levels and relation proposed by Swaisgood (2003) are used and the results of the FE model are compared to the database of case histories. In order to calculate the settlement ratio of the levee, the crest settlement is divided by the height of the levee plus the foundation (i.e. $\delta u_{v, c r e s t} / \mathrm{H}$ and the results are plotted as function of the maximum acceleration of the foundation bedrock (PGA), as shown in Figure 18). The results of the FE model are presented in Figure 19 and compared to the database of case histories. It is remarked that the response of the FE model follows the trend of the case histories only when no liquefaction or liquefaction in depth was observed (i.e. green points for the model with the liquefiable layer in depth) and moderate or minor level of damage is obtained. However, in case of soil liquefaction (red points for model with liquefiable layer close to surface), the levee experienced large settlements and its response is classified in the serious damage level. The importance of FE simulations in contrast to empirical methods is highlighted. Such a simulation can account for several aspects of soil behavior and better assess safety margins of structures.

Moreover, Swaisgood (2003) found that serious levels of damage were reported in instances where the PGA exceeded $0.2 \mathrm{~g}$ and as it can be seen in Figure 19 when PGA is greater than $0.2 \mathrm{~g}$, the results of the FE model are mostly classified in the serious damage range. However, it is noticed that a motion of $0.2 \mathrm{~g}$ provided the same crest settlement ratio (i.e. damage level) with a motion of $0.6 \mathrm{~g}$, which implies that not only the amplitude of a motion 


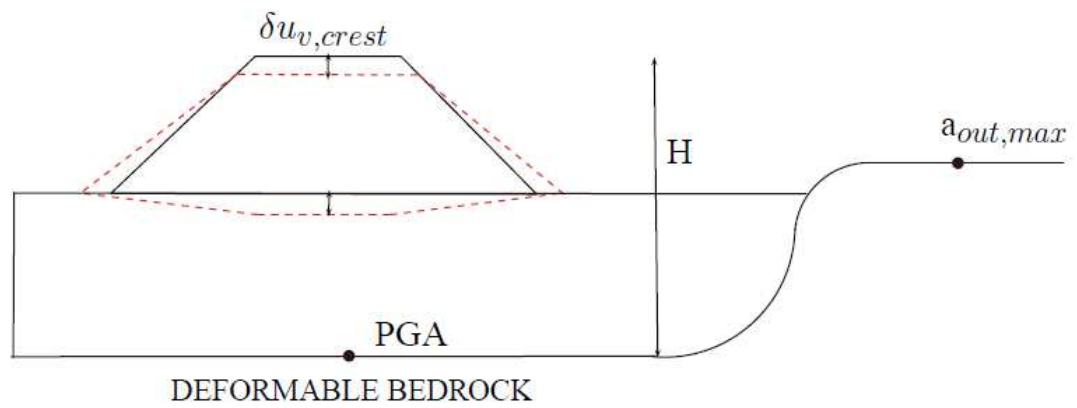

Fig. 18: Schematic representation of calculation of levee's settlements.

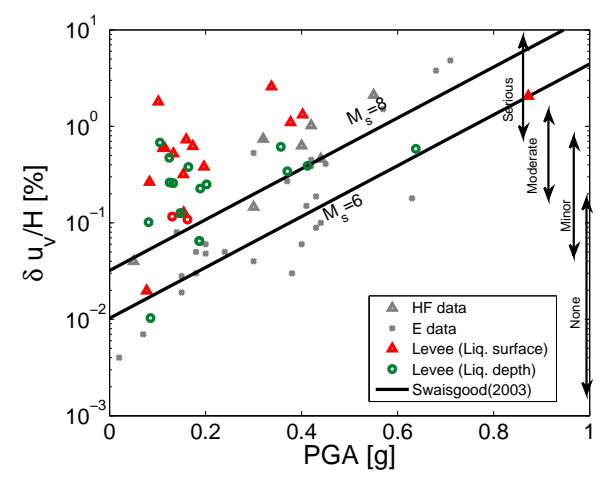

Fig. 19: Comparison of the crest settlement ratio of the FE model to the case history database.

is an important IM. For this reason, the propositions of Kawase (2011) are considered and the equivalent predominant frequency $\left(1 / T_{V / A}\right)$, the maximum velocity and acceleration of the ground motion are used as IMs. In Figure 20 the diagram of $\mathrm{a}_{b e d, \max }$ versus the equivalent predominant frequency is plotted for both models. In this diagram, equi- $\mathrm{v}_{b e d, \max }$ lines will be a slope from left-down side to right-up side, indicating uniform velocity of 10, 50, 100 and $200 \mathrm{~cm} / \mathrm{s}$. It is assumed that the intensity of the motion and consequently the 
severity of expected damages follow the direction of increasing velocity from the right-down corner towards the left-up one, as these motions will give high values of $\mathrm{a}_{b e d, \max }$ and $\mathrm{v}_{b e d, \max }$ at the same time. The red continuous line indicate an acceleration of $8 \mathrm{~m} / \mathrm{s}^{2}$ and the dashed red line uniform velocity of 100 $\mathrm{cm} / \mathrm{s}$. These lines are considered to be danger lines based on observations in Kobe, above which major damages are caused (Kawase, 2011). Based on these recommendations, the values of increasing crest settlements of the FE models follow the lines of increasing velocity, so it can be concluded that for the liquefaction vulnerability analysis the IM is a vector depending on the equivalent predominant frequency, the maximum acceleration and velocity of the input ground motion.

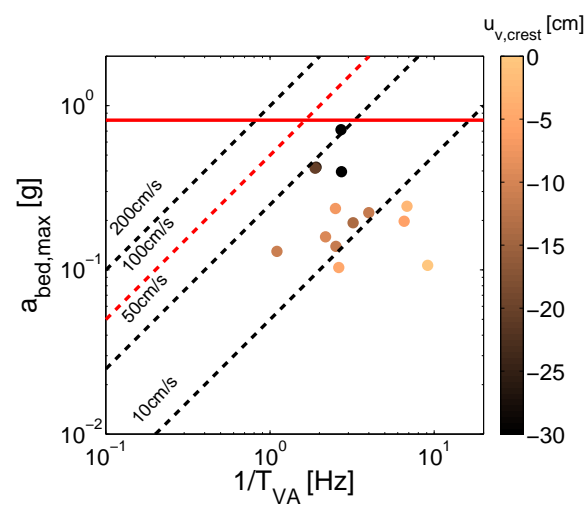

(a)

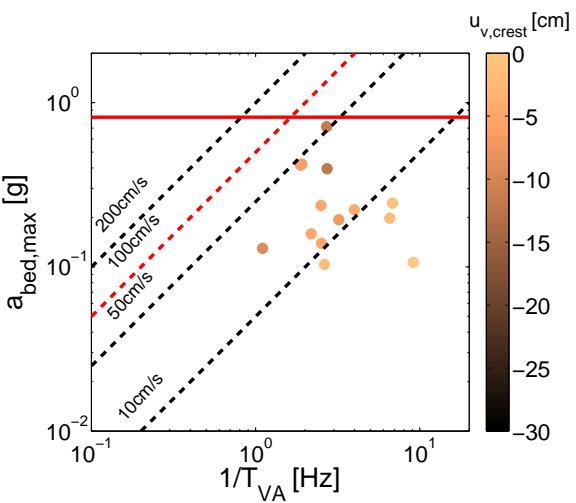

(b)

Fig. 20: Relation between input IMs for the considered EDP of interest (i.e. crest settlement): a) liquefiable layer close to surface, b) liquefiable layer in depth. 
Effect of input motion

In order to further evaluate the effect of the input ground motion, three earthquakes are chosen of different amplitudes: $\mathrm{a}_{b e d, \max }=0.14,0.24,0.42 \mathrm{~g}$ and duration of mainshock: $\mathrm{t}_{595}=7.3 \mathrm{~s}, 0.91 \mathrm{~s}, 4.3 \mathrm{~s}$. The moderate $\left(\mathrm{EQ} 2, \mathrm{a}_{\text {bed, } \max }=0.24 \mathrm{~g}\right)$ and strong motion $\left(\mathrm{EQ} 3, \mathrm{a}_{b e d, \max }=0.42 \mathrm{~g}\right)$ are those of Figures 5 and 12 , respectively, while the lower one is represented in Figure 21a (referred as EQ1 motion in Table 4). Furthermore, in Figure 21b the response spectra of the chosen motions are presented.

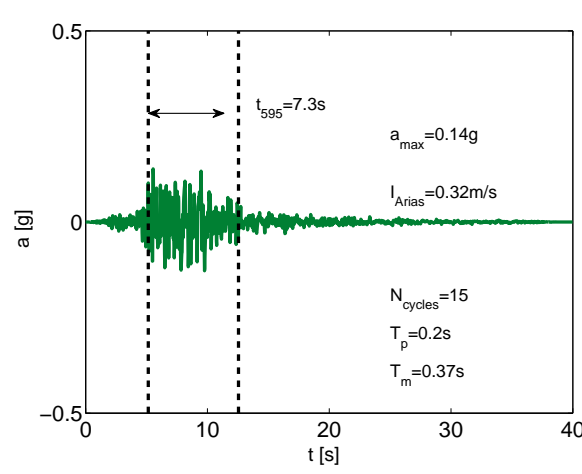

(a)

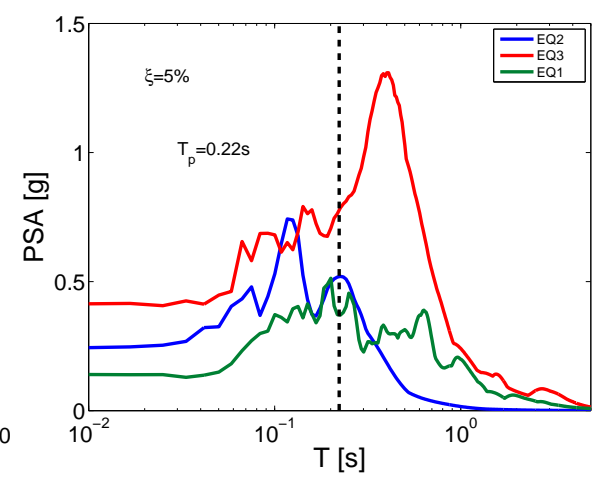

(b)

Fig. 21: Input ground motions: a) Low input motion (EQ1), d) Acceleration response spectra of three selected motions $(\xi=5 \%)$.

In Figure 22 the settlement at the end of each ground motion is presented. It is noticed that the low amplitude motion provides greater settlement at the crest and sides of the levee (Figure 22a) than the moderate (Figure 22b), due to the longer duration of mainshock $\left(t_{595}=7.3 \mathrm{~s}\right.$ for the low and $t_{595}=0.91 \mathrm{~s}$ 
for the moderate one) and consequently extended in time liquefaction of the foundation. Moreover, different failure mode is observed as the moderate motion implies a circular superficial failure mode in both sides of the levee, while the low one provides more extended settlements in the whole levee body. The failure mode associated to the low motion can be classified as Type 2, while that of the moderate as Type 1 and both of them refer to failure in the levee, according to Figure 2. Finally, in case of the strong motion, the whole foundation was liquefied, as shown in Figure 23, and as a consequence the whole levee settles down (Figure 22c) and is driven to complete collapse, i.e. failure Type 3 as indicated in Figure 2.

To further observe the failure path, the deviatoric strains are illustrated at the end of the motion for all cases in Figure 24. In the case of low motion (Figure 24a), two shear bands are generated in the liquefied region and merge inside the levee providing a diffuse zone of shear deformations. While the moderate motion provided two thinner shear bands that propagate towards the crest. Nevertheless in the case of strong motion, the shear deformations are more significant in the liquefied soil layer and a diffuse failure is observed.

According to the current results, it can be concluded that the important IMs are a combination of the equivalent predominant frequency, the maximum acceleration and velocity of the input ground motion. The duration of mainshock should also be considered as it has a strong effect on the extent of liquefaction. 


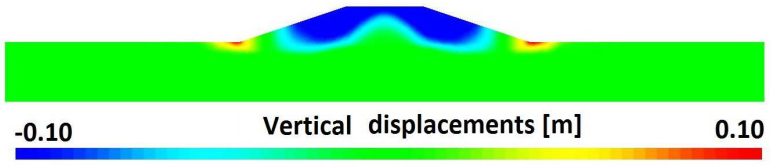

(a)

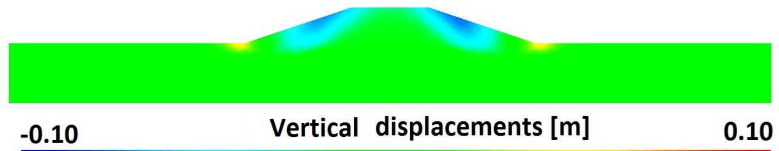

(b)

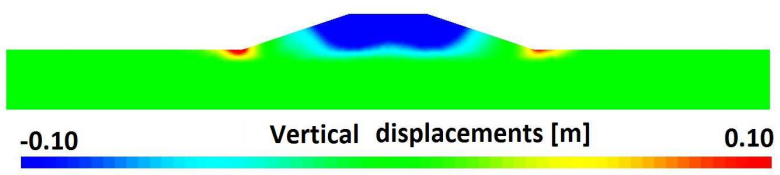

(c)

Fig. 22: Settlements at the end of the ground motion: a) Low (EQ1), b) Moderate (EQ2), c) Strong (EQ3).

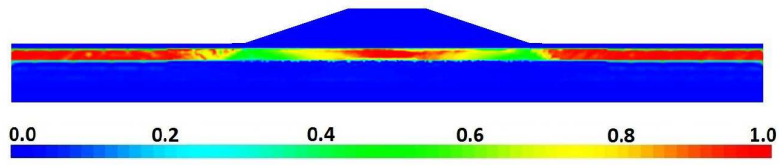

Fig. 23: Excess pore water pressure ratio $\left(r_{u}=\Delta p_{w} / \sigma_{o}^{\prime}\right)$ during the mainshock $(\mathrm{t}=5 \mathrm{~s})$ of the strong motion (EQ3). 


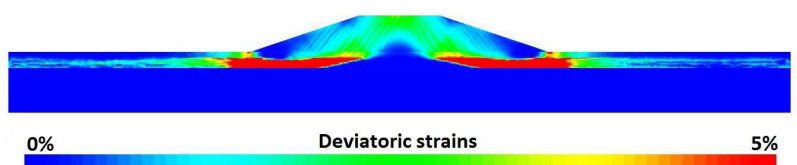

(a)

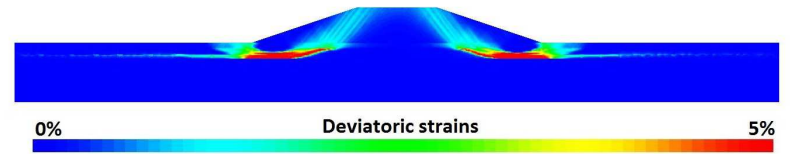

(b)

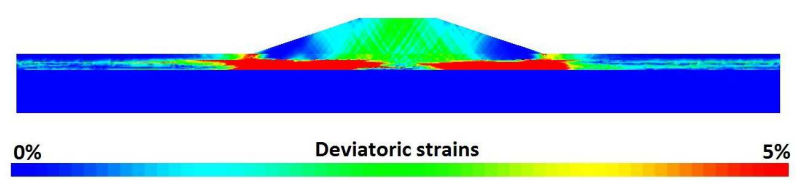

(c)

Fig. 24: Deviatoric strains $\varepsilon_{d}$ at the end of the ground motion: a) Low (EQ1), b) Moderate (EQ2), c) Strong (EQ3). 


\section{Conclusions}

Through a dynamic nonlinear FE analysis of a model of a levee founded on liquefiable soil substratum several aspects of the earthquake-induced failure mode of the structure were investigated. In particular, we analysed the specific capacities of several local scalar quantities, resulting from the FE transient solutions, to ascertain a safety factor assessing the stability of the earthen structure, remaining in the framework of the small-strain assumption and coupled hydromechanics framework. The first one is the relative distance to reach the critical state, $\mathrm{r}_{a p t}$ in $[0,1]$, based on the elastic-plastic soil local evolution, useful to analyse the onset of the localized and diffuse instabilities in the weakest areas of the soil domain, and playing as a residual strength criterion. The second one is the normalized second-order work density $\mathrm{d}^{2} \mathrm{~W}$ in $[-1,1]$, based on the soil effective stresses, which is a sufficient instability criterion if $\mathrm{d}^{2} \mathrm{~W}<0$ for the soil phase in the earthen structure.

Both quantities are computed from the soil state only (effective stresses contributing to the porous medium equilibrium), under an indirect hydromechanical coupling influence. We observed that these local quantities are able to exhibit very similar spatial distribution of critical zones in the earthen structure, at specific times during the transient analyses. Nevertheless, their temporal distributions differ greatly, as the time-evolutions of $\mathrm{d}^{2} \mathrm{~W}$ are very fast and its sign can change rapidly during the ground motion, while the $\mathrm{r}_{\text {apt }}$ quantity results from the internal state cumulative history of the soil material. Therefore it seems that a $\mathrm{r}_{a p t}$ based safety criterion is more relevant than a 
$\mathrm{d}^{2} \mathrm{~W}$ based one for dynamic applications, as it accounts for the soil loading history. The $\mathrm{r}_{a p t}$ distribution can be seen as a relevant local safety criterion for the soil material within the earthen structure after the seismic event.

In both models of varying depth of liquefiable foundation, liquefaction appears in the loose-to-medium sandy layer of the foundation. Due to higher values of stresses and soil's consolidation below the levee, less significant excess pore water pressure is observed. These results come to an agreement with the results obtained after centrifuge tests performed by Sharp and Adalier (2006).

In case of a liquefiable foundation close to the surface, a collapse surface is generated inside the liquefied region and extends towards the crest in both sides of the levee, accompanied by settlements and horizontal displacements. It is worth noting that a circular local instability at both sides of the levee is detected during the mainshock. However, the model reaches a stable state at the end of the ground motion, irrespective of the excess pore water pressure that may still appear in the foundation. This result discloses the deficiency of the second order work to take into account the accumulated co-seismic instability. For this reason, by calculating the soil's residual strength after the ground motion, low values of local safety factor (1.0-1.25) are found inside the liquefied layer in all models, denoting the significant degradation of the soil due to liquefaction. The earthquake-induced liquefaction led to soil degradation irrespective of its duration and it is apparent inside the levee, too. The use of soil's residual strength as an index of local safety factor highlights the capability of 
the model and of this indicator to account for the history of soil's behavior.

This indicator takes into consideration the loading history and can represent the evolution of soil's behavior, i.e. soil densification after liquefaction.

Through the liquefaction vulnerability analysis with several input ground motions, it is shown that the dynamic response of such structures is strongly related to the characteristics of the seismic ground motions and their importance of the seismic hazard in order to define the expected failure path is discussed. Each ground motion provided a different failure path and damage level. It is noticed that the important IMs are a combination of the equivalent predominant frequency, the maximum acceleration and velocity of the input ground motion. As a second step the duration of mainshock should also be considered, since it can be very severe in terms of structural damages. For instance a strong motion led to the extended liquefaction in the foundation part and the whole levee collapsed. Consequently, it is concluded that the failure mode of such structures depends on the interaction among the levee, foundation's liquefaction and characteristics of seismic ground motions.

To sum up, a collapse mechanism is identified for a levee-foundation system subjected to earthquake loading. Its crucial dependency on depth of the liquefiable layer, as well as, characteristics of the ground motion is demonstrated through parametric studies. It should be highlighted that the aforementioned conclusions are strictly limited to the current model subjected to the particular ground motions chosen. 
Acknowledgements

The research reported in this paper has been funded by the ANRT (Association Nationale de la Recherche et de la Technologie) under a CIFRE convention number 0120/2013 and is supported partially by the SEISM Paris Saclay Research Institute (http://www.institut-seism.fr/en/).

\section{A ECP elastoplastic constitutive model}

The model is written in the framework of the incremental plasticity, which assumes the decomposition of the total strain increment in two, elastic and plastic, parts. In what follows, a brief overview of the essential aspects of the constitutive model for primary loading paths is given. The elastic part is supposed to obey a non-linear elasticity behaviour, where the Young $(E)$ modulus is function of the mean effective stress $\left(p^{\prime}\right)$ :

$$
E=E_{\text {ref }}\left(\frac{p^{\prime}}{p_{\text {ref }}}\right)^{n_{e}}
$$

$E_{r e f}$ is the Young modulus measured at the mean reference pressure $\left(p_{r e f}\right)$. Adopting the soil mechanics sign convention (compression positive), the deviatoric primary yield surface of the $k$ plane is given by:

$$
f_{k}\left(\underline{\underline{\sigma}}^{\prime}, \varepsilon_{v}^{p}, r_{k}\right)=q_{k}-\sin \phi_{p p}^{\prime} \cdot p_{k}^{\prime} \cdot F_{k} \cdot r_{k}
$$

with:

$$
\begin{gathered}
F_{k}=1-b \ln \left(\frac{p_{k}^{\prime}}{p_{c}}\right) \\
p_{c}=p_{c o} \exp \left(\beta \varepsilon_{v}^{p}\right)
\end{gathered}
$$

where, $\underline{\underline{\sigma}}^{\prime}$ is the effective stress tensor and $\phi_{p p}^{\prime}$ is the friction angle at the critical state. The parameter $b$ controls the form of the yield surface in the $\left(p^{\prime}, q\right)$ plane and varies from $b=0$ to 1 passing from a Coulomb type surface to a Cam-Clay type one (Schofield and Wroth, 
1968). $\beta$ is the plasticity compression modulus and $p_{c o}$ represents the critical state stress corresponding to the initial voids ratio.

The internal variable $r_{k}$, called degree of mobilized friction, is associated with the plastic deviatoric strain. This variable introduces the effect of shear hardening of the soil and permits the decomposition of the behaviour domain into pseudo-elastic, hysteretic and mobilized domains. It is given by:

$$
r_{k}=r_{k}^{e l}+\frac{\left|\bar{\varepsilon}^{p}-\bar{\varepsilon}_{o}^{p}\right|}{a+\left|\bar{\varepsilon}^{p}-\bar{\varepsilon}_{o}^{p}\right|}
$$

$\bar{\varepsilon}^{p}$ is the plastic shear strain accumulated during the shearing $\left(\bar{\varepsilon}^{p}=\int_{t o}^{t} \dot{\bar{\varepsilon}}^{p} d t\right) ; \bar{\varepsilon}_{o}^{p}$ is the plastic shear strain accumulated from the very beginning of the loading until the last unloading $\left(\bar{\varepsilon}_{o}^{p}=\int_{0}^{t o} \dot{\bar{\varepsilon}}^{p} d t\right)$. Parameter $a$ is defined as :

$$
a=a_{1}+\left(a_{2}-a_{1}\right) \alpha_{k}\left(r_{k}\right)
$$

where :

$$
\begin{array}{ll}
\alpha_{k}=0 & \text { if } r_{k}^{\text {elas }}<r_{k}<r_{k}^{\text {hys }} \\
\alpha_{k}=\left(\frac{r_{k}-r_{k}^{h y s}}{r_{k}^{\text {mob }}-r_{k}^{h y s}}\right)^{m} & \text { if } r_{k}^{\text {hys }}<r<r_{k}^{\text {mob }} \\
\alpha_{k}=1 & \text { if } r_{k}^{\text {mob }}<r_{k}<1
\end{array}
$$

$a_{1}, a_{2}$ and $m$ are model parameters and $r^{h y s}$ and $r^{m o b}$ designate the extend of the domain where hysteresis degradation occurs. When the plastic strains grow dramatically in the soil, the function $r_{k}$ reaches its maximal value asymptotically:

$$
\lim _{\bar{\varepsilon} p \rightarrow+\infty} r_{k}=1 \text { or } \lim _{\bar{\varepsilon} p \rightarrow+\infty} q_{k}=\sin \phi_{p p}^{\prime} \cdot p_{k}^{\prime} \cdot F_{k}
$$

The isotropic yield surface is assumed to be :

$$
f_{\text {iso }}=\left|p^{\prime}\right|-d p_{c} r_{i s o}
$$

with :

$$
r_{i s o}=r_{i s o}^{e l a}+\frac{\int_{0}^{t}\left|\left(\dot{\varepsilon}_{v}^{p}\right)_{i s o}\right| d t}{c \frac{p_{c}}{p_{r e f}}+\int_{0}^{t}\left|\left(\dot{\varepsilon}_{v}^{p}\right)_{i s o}\right| d t}
$$

where $d$ is a model parameter representing the distance between the isotropic consolidation line and the critical state line in the $\left(\varepsilon_{v}-\ln p^{\prime}\right)$ plane (i.e. $\left.d=\left|p_{i s o}^{\prime}\right| / p_{c}\right)$ and $c$ controls the 
volumetric hardening. In the model, an associated flow rule in the deviatoric plane $(k)$ is assumed, and the Roscoe's dilatancy law (Roscoe et al, 1958) is used to obtain the increment of the volumetric plastic strain of each deviatoric mechanism so that :

$$
\dot{\varepsilon}_{v k}^{p}=\dot{\lambda}_{k}^{p} \cdot \alpha_{\psi} \cdot \alpha_{k}\left(r_{k}\right)\left(\sin \psi-\frac{q_{k}}{p_{k}^{\prime}}\right)
$$

where $\psi$ is the characteristic angle (Luong, 1980) defining the limit between dilatancy and contractance of the material and $\alpha_{\psi}$ a constant parameter.

\section{B Soil parameters}


Table 3: ECP model's parameters for the soil.

\begin{tabular}{|c|c|c|c|}
\hline Parameter & LMS & $\begin{array}{l}\text { Dense sand } \\
\text { (Foundation) }\end{array}$ & $\begin{array}{l}\text { Dense sand } \\
\text { (Levee) }\end{array}$ \\
\hline$\rho\left[\mathrm{kg} / \mathrm{m}^{3}\right]$ : Soid density & 1755 & 1755 & 1755 \\
\hline$\nu[1]$ : Poisson's ratio & 0.3 & 0.4 & 0.15 \\
\hline$K_{0}[1]$ : Coefficient of lateral earth pressure & 0.7 & 0.7 & 0.7 \\
\hline \multicolumn{4}{|l|}{ Elasticity } \\
\hline$E_{\text {ref }}[\mathrm{MPa}]$ : Young's modulus & 754 & 1850 & 1457 \\
\hline$n_{e}[1]$ : Nonlinear degree & 0.5 & 0.47 & 0.6 \\
\hline$p_{r e f}^{\prime}[\mathrm{MPa}]$ : Reference mean stress & 1.0 & 1.0 & 1.0 \\
\hline \multicolumn{4}{|c|}{ Critical State and Plasticity } \\
\hline$\beta[1]$ : Plastic compressibility modulus & 33 & 44 & 43 \\
\hline$b[1]$ : Yield surface shape & 0.12 & 0.8 & 0.23 \\
\hline$d[1]$ : Isotropic consolidation distance & 2.0 & 5.0 & 10.0 \\
\hline$\phi_{p p}^{\prime}\left[{ }^{\circ}\right]$ : Friction angle & 30 & 37 & 36 \\
\hline$p_{c 0}[\mathrm{MPa}]$ : Initial critical stress & $40 \cdot 10^{-3}$ & 0.4 & 1.2 \\
\hline \multicolumn{4}{|c|}{ Flow Rule and Hardening } \\
\hline$a_{1}[1]$ : Primary plastic stiffness & 0.0001 & 0.0002 & 0.0001 \\
\hline$a_{2}[1]$ : Secondary plastic stiffness & 0.005 & 0.0004 & 0.01 \\
\hline$c^{m}[1]:$ Monotonic isotropic hardening & 0.004 & 0.01 & 0.2 \\
\hline$c^{c}[1]$ : Cyclic isotropic hardening & 0.002 & 0.005 & 0.1 \\
\hline$\left.\psi{ }^{[0}\right]$ : Characteristic angle & 30 & 37 & 36 \\
\hline$\alpha_{\psi}[1]$ : Volumetric parameter & 1.0 & 1.0 & 1.0 \\
\hline$m[1]:$ Cyclic loading exponential & 1.5 & 1.0 & 1.0 \\
\hline \multicolumn{4}{|c|}{ Threshold Domains } \\
\hline$r_{d}^{\text {ela }}[1]$ : Deviatoric elastic & 0.03 & 0.005 & 0.001 \\
\hline$r_{i s o}^{e l a}[1]$ : Isotropic elastic & 0.001 & 0.0001 & 0.0001 \\
\hline$r_{d}^{c y c}[1]:$ Cyclic deviatoric & 0.03 & 0.005 & 0.001 \\
\hline$r_{i s o}^{c y c}[1]$ : Cyclic isotropic & 0.001 & 0.0001 & 0.0001 \\
\hline$r^{\text {hys }}[1]$ : Hysteretic & 0.04 & 0.04 & 0.01 \\
\hline$r^{\text {mob }[1] \text { : Mobilized }}$ & 0.8 & 0.9 & 0.9 \\
\hline
\end{tabular}




\section{Input ground motions}

Table 4: Properties of input seismic motions.

\begin{tabular}{|c|c|c|c|c|c|c|c|c|}
\hline Set $^{*}$ & Event & Year & Station & Record/Component & $\mathrm{ID}^{* *}$ & $\mathbf{M}_{w}$ & $\mathbf{R}^{* * *}[\mathrm{~km}]$ & $\mathbf{V}_{s, 30}[\mathrm{~m} / \mathrm{s}]$ \\
\hline EQ1 & Northridge-01 01-17 & 1994 & Vasquez Rocks Park & NORTHR_VAS090 & 1091 & 6.69 & 23.1 & 966 \\
\hline EQ2 & Emilia-Romagna 05-29 7:00 & 2012 & SAN0 & HNE & IT-2012-0011 & 6.0 & 10.2 & EC8 $\mathrm{C}^{*}$ (inside building) \\
\hline EQ3 & Northridge-01 01-17 & 1994 & Pacoima Dam (downstr) & NORTHR_PAC175 & 1050 & 6.69 & 4.92 & 2016 \\
\hline EQ4 & Imperial Valley 10-15 23:16 & 1979 & Compuertas & IMPVALL_H-CMP015 & 167 & 6.53 & 13.52 & 260 \\
\hline EQ5 & Landers 06-28 11:58 & 1992 & North Palm Springs & LANDERS_NPS000 & 882 & 7.28 & 26.84 & 345 \\
\hline EQ6 & Loma Prieta 10-18 00:05 & 1989 & WAHO & LOMAP_WAHO00 & 811 & 6.93 & 11.03 & 388 \\
\hline EQ7 & Coyote Lake 08-06 & 1979 & Gilroy Array \# 6 & COYOTELK_G06230 & 150 & 5.74 & 0.42 & 663 \\
\hline EQ8 & Hawaii 01-18 12:17 & 2010 & Chan00 & EW & - & 4.3 & - & - \\
\hline EQ9 & Friuli, Italy-01 05-06 20:00 & 1976 & Tolmezzo & FRIULI.A_A-TMZ000 & 125 & 6.5 & 14.97 & 505 \\
\hline EQ10 & San Fernando 02-09 & 1971 & Lake Hughes \#4 & SFERN_L04111 & 72 & 6.61 & 19.45 & 600 \\
\hline EQ11 & Northridge-01 01-17 & 1994 & LA-Wonderland Ave & NORTHR_WON095 & 1011 & 6.69 & 15.11 & 1222 \\
\hline EQ12 & Northridge-01 01-17 & 1994 & LA-Wonderland Ave & NORTHR_WON185 & 1011 & 6.69 & 15.11 & 1222 \\
\hline EQ13 & Irpinia, Italy-01 11-23 & 1980 & Bagnoli Irpinio & ITALY_A-BAG000 & 285 & 6.9 & 8.14 & 650 \\
\hline EQ14 & Morgan Hill 04-24 & 1984 & Coyote Lake Dam - Southwest Abutment & MORGAN_CYC195 & 451 & 6.19 & 0.18 & 561 \\
\hline EQ15 & Morgan Hill 04-24 & 1984 & Gilroy Array \# 6 & MORGAN_G06000 & 459 & 6.19 & 9.85 & 663 \\
\hline
\end{tabular}

* The "a" and "b" refer to the fact that one horizontal component is used in "a" and the other in "b"

** ID as given on the NGA database (http://ngawest2.berkeley.edu)

*** Joyner-Boore source-to-site distance.

\section{References}

Adalier K, Sharp MK (2004) Dynamic behavior of embankment dam on liquefiable foundation subject to moderate earthquake loading. In: 13th World Conference on Earthquake Engineering, Vancouver, B.C., Canada, 1025

Andrade JE, Ramos AM, Lizcano A (2013) Criterion for flow liquefaction instability. Acta

Geotechnica DOI 10.1007/s11440-013-0223-x 
Aubry D, Modaressi H (1990) Dynamic Analysis of Saturated Non Linear Media. In: Numerical Methods and Constitutive Modelling in Geomechanics, International Centre for Mechanical Sciences, vol 311, Springer-Verlag Wien, pp 211-235

Aydingun O, Adalier K (2003) Numerical analysis of seismically induced liquefaction in earth embankment foundations. Part I. Benchmark model. Canadian Geotechnical Journal $40(4): 753-765$

Baker JW (2007) Quantitative Classification of Near-Fault Ground Motions Using Wavelet Analysis. Bulletin of the Seismological Society of America 97(5):1486-1501, DOI $10.1785 / 0120060255$

Baker JW, Lin T, Shahi SK, Jayaram N (2011) New Ground Motion Selection Procedures and Selected Motions for the PEER Transportation Research Program. Tech. rep., PEER Report 2011, Pacific Earthquake Engineering Research Center. College of Engineering. University of California, Berkeley

Bardet JP (1997) Experimental Soil Mechanics. Prentice-Hall

Bigoni D, Hueckel T (1991) Uniqueness and localization-I. Associative and non-associative elastoplasticity. International Journal of Solids and Structures 28(2):197-213

Borja RI (2006) Condition for liquefaction instability in fluid-saturated granular soils. Acta Geotechnica 1:211-224, DOI 10.1007/s11440-006-0017-5

Buscarnera G, di Prisco C (2012) Discussing the definition of the second-order work for unsaturated soils. International Journal for Numerical and Analytical Methods in Geomechanics 36(December 2010):36-49, DOI 10.1002/nag

Byrne PM, Park Ss, Beaty M, Sharp M, Gonzalez L, Abdoun T (2004) Numerical modeling of liquefaction and comparison with centrifuge tests. Canadian Geotechnical Journal 211:193-211, DOI 10.1139/T03-088

Coelho P, Haigh SK, Madabhushi SPG (2004) Centrifuge modelling of earthquake effects in unifrom deposits of saturated sand. In: 5th International Conference on Case Histories in Geotechnical Engineering, New York, April 13-17, 36

Costa D'Aguiar S, Modaressi-Farahmand-Razavi A, Dos Santos J, Lopez-caballero F (2011)

Elastoplastic constitutive modelling of soil-structure interfaces under monotonic and 
cyclic loading. Computers and Geotechnics 38(4):430-447

Coussy O (1991) Mecanique des milieux poreux, technip edn

Darve F, Laouafa F (2000) Instabilities in granular materials and application to landslides. Mechanics of Cohesive-Frictional Materials 5(8):627-652

Foucault A (2009) Loi de comportement cyclique de Hujeux pour les sols. Documentation of Code_Aster [R70123]

Granet S (2015) Modèles de comportement THHM. Documentation of Code_Aster [R70111]

Hamadi K, Modaressi-Farahmand Razavi A, Darve F (2008) Bifurcation and instability modelling by a multimechanism elasto-plastic model. International Journal for Numerical and Analytical Methods in Geomechanics 32:461-492, DOI 10.1002/nag

Hill R (1958) A general theory of uniqueness and stability in elastic-plastic solids. Journal of the Mechanics and Physics of Solids 6:236-249

Hughes T (2000) The Finite Element Method: Linear Static and DynamicFinite Element Analysis, dover edit edn. DOVER Publications

Hujeux JC (1985) Une loi de comportement pour le chargement cyclique des sols. Presses ENPC pp 278-302

Iervolino I, Cornell CA (2005) Record selection for nonlinear seismic analysis of structures. Earthquake Spectra 21(3):685-713

Ishikawa H, Saito K, Nakagawa K, Uzuoka R (2015) Liquefaction analysis of a damaged river levee during the 2011 Tohoku earthquake. In: 14th International Conference of the International Association for Computer Methods and Advances in Geomechanics, Kyoto, Japan, pp 673-677

Kawase H (2011) Strong motion characteristics and their damage impact to structures during the Off Pacific Coast of Tohoku earthquake of March 11, 2011; How extraordinary was this M9.0 earthquake? In: 4th IASPEI / IAEE International Symposium: Effects of Surface Geology on Seismic Motion, University of California Santa Barbara

Kim MK, Lee SH, Choo YW, Kim DS (2011) Seismic behaviors of earth-core and concretefaced rock-fill dams by dynamic centrifuge tests. Soil Dynamics and Earthquake Engineering 31:1579-1593 
Kontoe S, Zdravkovic L, Potts D (2008) An assessment of time integration schemes for dynamic geotechnical problems. Computers and Geotechnics 35(2):253-264

Koutsourelakis S, Prévost J H, Deodatis G (2002) Risk assessment of an interacting structure-soil system due to liquefaction. Earthquake Engineering and Structural Dynamics 31(4):851-879.

Kuhl D, Crisfield M (1999) Energy-conserving and decaying algorithms in non-linear structural dynamics. International Journal for numerical methods in engineering 45:569-599

Lade P (1994) Instability and liquefaction of granular materials. Computers and Geotechnics $16: 123-151$

Lanzo G, Pagliaroli A (2012) Seismic site effects at near-fault strong-motion stations along the Aterno River Valley during the $\mathrm{Mw}=6.32009$ L'Aquila earthquake. Soil Dynamics and Earthquake Engineering 40:1-14

Lopez-Caballero, F and Modaressi-Farahmand-Razavi, A (2008) Numerical simulation of liquefaction effects on seismic SSI. Soil Dynamics and Earthquake Engineering 28(2):8598.

Lopez-Caballero F, Modaressi-Farahmand-Razavi A (2013) Numerical simulation of mitigation of liquefaction seismic risk by preloading and its effects on the performance of structures. Soil Dynamics and Earthquake Engineering 49:27-38

Lopez-Caballero F, Modaressi-Farahmand-Razavi A, Modaressi H (2007) Nonlinear numerical method for earthquake site response analysis I-elastoplastic cyclic model and parameter identification strategy. Bulletin of Earthquake Engineering 5:303-323, DOI $10.1007 / \mathrm{s} 10518-007-9032-7$

Lopez-Caballero F, Modaressi A, Stamatopoulos C (2016) Numerical evaluation of earthquake settlements of road embankments and mitigation by preloading. International Journal of Geomechanics 16(5):C4015006

Lu C W, Oka F, Zhang F (2008) Analysis of soil-pile-structure interaction in a two-layer ground during earthquakes considering liquefaction. International Journal for Numerical and Analytical Methods in Geomechanics 32(8):863-895. 
Luong MP (1980) Phénomènes cycliques dans les sols pulvérulents. Revue Française de Géotechnique 10(1):39-53

Maharjan M, Takahashi A (2014) Liquefaction-induced deformation of earthen embankments on non-homogeneous soil deposits under sequential ground motions. Soil Dynamics and Earthquake Engineering 66:113-124, DOI 10.1016/j.soildyn.2014.06.024

Modaressi H (1987) Modélisation numérique de la propagation des ondes dans les milieux poreux anélastiques. PhD thesis, Ecole Centrale Paris

Modaressi H, Benzenati I (1994) Paraxial approximation for poroelastic media. Soil Dynamics and Earthquake Engineering 13(2):117-129

Mohammadnejad T, Andrade JE (2015) Flow liquefaction instability prediction using finite elements. Acta Geotechnica 10(1):83-100

Montoya-Noguera S, Lopez-Caballero F (2016) Effect of coupling excess pore pressure and deformation on nonlinear seismic soil response. Acta Geotechnica 11(1):191-207.

Najma A, Latifi M (2017) Predicting flow liquefaction, a constitutive model approach Acta Geotechnica 12(1):793-808

NAVFAC (1982) DESIGN MANUAL 7.01: Soil Mechanics. Tech. rep., Naval Facilities Engineering Command, Alexandria, Virginia

Nguyen TD (2006) Mod\{é\}lisation du comportement des mat\{é\}riaux granulaires. Application aux barrages en terre. PhD thesis, Ecole Centrale Paris

Oka F, Yashima A, Shibata T, Kato M, Uzuoka R (1994) FEM-FDM Coupled Liquefaction Analysis of a Porous Soil Using an Elasto-Plastic Model. Applied Scientific Research $52: 209-245$

Oka F, Tsai P, Kimoto S, Kato R (2012) Damage patterns of river embankments due to the 2011 off the Pacific Coast of Tohoku Earthquake and a numerical modeling of the deformation of river embankments with a clayey subsoil layer. Soils and Foundations 52(5):890-909, DOI 10.1016/j.sandf.2012.11.010

Okamura M, Tamamura S, Yamamoto R (2013) Seismic stability of embankments subjected to pre-deformation due to foundation consolidation. Soils and Foundations 53(1):11-22 
Ozutsumi O, Sawada S, Iai S, Takeshima Y, Sugiyama W, Shimazu T (2002) Effective stress analyses of liquefaction-induced deformation in river dikes. Soil Dynamics and Earthquake Engineering 22(9-12):1075-1082

Popescu R, Prévost J H, Deodatis G, Chakrabortty P (2006) Dynamics of nonlinear porous media with applications to soil liquefaction. Soil Dynamics and Earthquake Engineering $26(6-7): 648-665$

Porter KA (2003) An Overview of PEER's Performance-Based Earthquake Engineering Methodology. In: Ninth International Conference on Applications of Statistics and Probability in Civil Engineering (ICASP9) July 6-9, 2003, San Francisco

Roscoe KH, Schofield AN, Wroth CP (1958) On the yielding of soils. Géotechnique 8(1):2252

Ruiz S, Saragoni GR (2009) Free vibration of soils during large earthquakes. Soil Dynamics and Earthquake Engineering 29:1-16, DOI 10.1016/j.soildyn.2008.01.005

Sadeghi H, Kimoto S, Oka F, Shahbodagh B (2014) Dynamic analysis of river embankments during earthquakes using a finite deformation FE analysis method. In: 14th International Conference of the International Association for Computer Methods and Advances in Geomechanics, Kyoto, Japan, 2011, pp 637-642

Saez E (2009) Dynamic nonlinear soil-structure interaction. PhD thesis, Ecole Centrale Paris

Sasaki Y, Tamura K (2007) Failure mode of embankments due to recent earthquakes in japan. In: 4th International Conference on Earthquake Geotechnical Engineering, Thessaloniki, Greece, 1479

Schofield AN and Wroth CP (1968) Critical State Soil Mechanics. McGraw-Hill, London

Seed HB, Idriss IM (1970) Soil moduli and damping factors for dynamic response analysis. Tech. rep., University of California, Berkeley

Sharp MK, Adalier K (2006) Seismic response of earth dam with varying depth of liquefiable foundation layer. Soil Dynamics and Earthquake Engineering 26:1028-1037

Singh R, Roy D, Jain SK (2005) Analysis of earth dams affected by the 2001 Bhuj Earthquake. Engineering Geology 80(3-4):282-291 
Stamatopoulos C, Aneroussis S (2004) Sliding-block back analyses of liquefaction-induced slides. In: 13th World Conference on Earthquake Engineering, Vancouver, B.C., Canada, August 1-6, 2004, 3209

Swaisgood JR (2003) Embankment dam deformation caused by earthquakes. In: Pacific Conference on Earthquake Engineering

Terzaghi K, Peck RB (1967) Soil mechanics in engineering practice. Wiley

Xia Zf, Ye Gl, Wang Jh, Ye B, Zhang F (2010) Fully coupled numerical analysis of repeated shake-consolidation process of earth embankment on liquefiable foundation. Soil Dynamics and Earthquake Engineering 30(11):1309-1318, DOI 10.1016/j.soildyn.2010.06.003

Zhang JM, Wang G (2012) Large post-liquefaction deformation of sand, part I: physical mechanism, constitutive description and numerical algorithm. Acta Geotechnica 7:69113.

Zienkiewicz O C, Chang C T, Bettess P (1980) Drained, undrained, consolidating and dynamic behaviour assumptions in soils. Géotechnique 30(4):385-395.

Zienkiewicz OC, Chan AHC, Pastor M, Paul DK, Shiomi T (1990) Static and Dynamic Behaviour of Soils: A Rational Approach to Quantitative Solutions. I. Fully Saturated Problems. In: Royal Society of London. Series A, Mathematical and Physical Sciences, June, pp 285-309, DOI 10.1098/rspa.1990.0061 